JOURNAL OF

SYMPLECTIC GEOMETRY

Volume 3, Number 3, 357-383, 2005

\title{
ON CERTAIN SYMPLECTIC CIRCLE ACTIONS
}

\author{
LEONOR GODINHO
}

In this work we use localization formulas in equivariant cohomology to show that some symplectic actions on $\mathbf{6}$-dimensional manifolds with a finite fixed point set must be Hamiltonian. Moreover, we show that their fixed point data (number of fixed points and their isotropy weights) is the same as in $\boldsymbol{S}^{\mathbf{2}} \times \boldsymbol{S}^{\mathbf{2}} \times \boldsymbol{S}^{\mathbf{2}}$ equipped with a diagonal circle action, and we compute their cohomology rings.

\section{Introduction}

A circle action on a compact symplectic manifold $M$ is symplectic if it preserves the symplectic form. In addition, it is Hamiltonian if its generating vector field $X$ is Hamiltonian that is, if it satisfies $\iota_{X} \omega=d H$ where $H \in$ $C^{\infty}(M)$ is the Hamiltonian function.

An obvious necessary condition for a circle action to be Hamiltonian is to have fixed points, which correspond to the critical points of $H$. For Kähler manifolds and more generally for manifolds of Lefschetz type, this condition is also sufficient (cf. [MD-S]). Moreover, McDuff proved in [MD] that this result holds for all four-dimensional manifolds. However, this is not true for higher dimensions. In fact, McDuff constructed in [MD], a six-dimensional manifold equipped with a non-Hamiltonian symplectic circle action with fixed points. Hence, for higher dimensions, we need more conditions, either on the manifold or on the action, to make sure it is Hamiltonian.

One possible conjecture is that a symplectic action with isolated fixed points must be Hamiltonian. This result has already been proved in [T-W1] by Tolman and Weitsman, in the case of a semi-free action that is, free outside the fixed point set. Moreover, there are no known counterexamples (the fixed point sets in McDuff's six-dimensional example are tori). The argument used by Tolman and Weitsman uses integration in equivariant cohomology. Nevertheless, even though it generalizes to the case of nonsemifree circle actions, it fails to eliminate all non-Hamiltonian examples with a non-empty fixed point set. 
Trying to obtain more information on this problem, we use equivariant cohomology localization theorems as well as some topological conditions, to obtain additional information in the six-dimensional case of circle actions with isolated fixed points. Indeed, we prove the following theorems for circle actions for which the isotropy weights on the normal bundles of each fixed point are always $( \pm n, \pm m, \pm k)$ for positive integers $n \geq m \geq k \geq 1$ such that $n \neq m+k$ :

Theorem 1.1. Let $S^{1}$ act on a six-dimensional symplectic, compact connected manifold. If all fixed points are isolated and satisfy the condition that on their normal bundles the isotropy weights are always $n \geq m \geq k$, where $n \neq m+k$, then if the fixed point set $\operatorname{Fix}_{S^{1}}(M)$ is non-empty, the action must be Hamiltonian.

Theorem 1.2. If the conditions of Theorem 1.1 hold, then the number of fixed points with a given set of isotropy weights $( \pm n, \pm m, \pm k)$ is the same as in a product of spheres $S^{2} \times S^{2} \times S^{2}$ equipped with a diagonal circle action which rotates each sphere at speed $n, m$ and $k$.

Finally, using the above theorems, we show that any such space for which $n>m>k>2$ are pairwise relatively prime, has the same cohomology and Chern classes of either the product of spheres equipped with a diagonal action rotating each sphere at speed $n, m$ and $k$, or the $S^{1}$-space $E$ (also an $S^{2} \times S^{2}$-bundle over $S^{2}$ ) described in Section 4 . Indeed, we have the following theorem:

Theorem 1.3. If the conditions of Theorems 1.1 and 1.2 hold and, in addition, $n>m>k>2$ are pairwise relatively prime then, there either exists a map from $\operatorname{Fix}_{S^{1}}(M)$ to $\operatorname{Fix}_{S^{1}}\left(\left(S^{2}\right)^{3}\right)$ (the fixed point set of a diagonal circle action on the product of three spheres rotating each one at speeds $n, m, k$ ) or a map from $\operatorname{Fix}_{S^{1}}(M)$ to $\operatorname{Fix}_{S^{1}}(E)$ (the fixed point set of the $S^{1}$ space $E$ described in Section 4$)$ which respectively identifies the restrictions $i^{*}$ and $j^{*}$ or $i^{*}$ and $\tilde{j}^{*}$, of the equivariant cohomology classes to the fixed point sets, where

$$
\begin{array}{ll}
i^{*}: & H_{S^{1}}^{*}(M, \mathbf{Z}) \longrightarrow H_{S^{1}}^{*}\left(\operatorname{Fix}_{S^{1}}(M), \mathbf{Z}\right) \\
j^{*}: & H_{S^{1}}^{*}\left(\left(S^{2}\right)^{3}, \mathbf{Z}\right) \longrightarrow H_{S^{1}}^{*}\left(\operatorname{Fix}_{S^{1}}\left(\left(S^{2}\right)^{3}\right), \mathbf{Z}\right) \\
\tilde{j}^{*}: & H_{S^{1}}^{*}(E, \mathbf{Z}) \longrightarrow H_{S^{1}}^{*}\left(\operatorname{Fix}_{S^{1}}(E), \mathbf{Z}\right)
\end{array}
$$

are induced by the inclusions $i: \operatorname{Fix}_{S^{1}}(M) \rightarrow M, j: \operatorname{Fix}_{S^{1}}\left(\left(S^{2}\right)^{3}\right) \rightarrow\left(S^{2}\right)^{3}$ and $\tilde{j}: \operatorname{Fix}_{S^{1}}(E) \rightarrow E$. Moreover, these maps send the images of equivariant Chern classes of $M$ to those of $S^{2} \times S^{2} \times S^{2}$ or $E$.

Then, since by a Theorem of Kirwan $[\mathbf{K i}]$, the maps $i^{*}$ and $j^{*}$ above are injections, Theorem 1.3 implies that there is either an isomorphism between 
$H_{S^{1}}^{*}(M, \mathbf{Z})$ and $H_{S^{1}}^{*}\left(\left(S^{2}\right)^{3}, \mathbf{Z}\right)$, in which case the equivariant cohomology ring is

$$
H_{S^{1}}^{*}(M, \mathbf{Z})=\frac{\mathbf{Z}\left[a_{1}, a_{2}, a_{3}, y\right]}{\left(w_{i} a_{i} y-a_{i}^{2}\right)}
$$

or between $H_{S^{1}}^{*}(M, \mathbf{Z})$ and $H_{S^{1}}^{*}(E, \mathbf{Z})$, in which case the equivariant cohomology ring is

$$
H_{S^{1}}^{*}(M, \mathbf{Z})=\frac{\mathbf{Z}\left[a_{1}, a_{2}, a_{3}, y\right]}{\left(a_{1}\left(w_{1} y+e a_{3}-a_{1}\right), a_{2}\left(w_{2} y-e a_{3}-a_{2}\right), a_{3}\left(w_{3} y-a_{3}\right)\right)},
$$

where the $w_{i}$ 's are the negative isotropy weights of the fixed points of index 2 corresponding to the generating classes $a_{i}$ (cf. Section 5 ), and $e \in \mathbf{Z}$ is such that $\omega_{2}=\omega_{1}+e \omega_{3}$. In the first case, the equivariant Chern series $c_{t}(M)=\sum_{i} t^{i} c_{i}(M)$ is given by

$$
c_{t}(M)=\Pi_{i=1}^{3}\left(1+t\left(2 a_{i}-w_{i} y\right)\right)
$$

and, in the second one, it is given by

$c_{t}(M)=\left(1+t\left(2 a_{1}-\omega_{1} y-e a_{3}\right)\right)\left(1+t\left(2 a_{2}-\omega_{2} y+e a_{3}\right)\right)\left(1+t\left(2 a_{3}-\omega_{3} y\right)\right)$.

Moreover, in the first case there is an isomorphism between $H^{*}(M, \mathbf{Z})$ and $H^{*}\left(\left(S^{2}\right)^{3}, \mathbf{Z}\right)$ taking Chern classes to Chern classes and so the ordinary cohomology ring is given by

$$
H^{*}(M, \mathbf{Z})=\frac{\mathbf{Z}\left[a_{1}, a_{1}, a_{3}\right]}{\left(a_{i}^{2}\right)}
$$

and the Chern series is $c_{t}(M)=\Pi_{i=1}^{3}\left(1+t 2 a_{i}\right)$. On the second case, there is an isomorphism between $H^{*}(M, \mathbf{Z})$ and $H^{*}(E, \mathbf{Z})$, and so the ordinary cohomology ring is

$$
H^{*}(M, \mathbf{Z})=\frac{\mathbf{Z}\left[a_{1}, a_{1}, a_{3}\right]}{\left(a_{1}\left(e a_{3}-a_{1}\right), a_{2}\left(e a_{3}+a_{2}\right), a_{3}^{2}\right)}
$$

and the Chern series is $c_{t}(M)=\left(1+t\left(2 a_{1}-e a_{3}\right)\right)\left(1+t\left(2 a_{2}+e a_{3}\right)\right)\left(1+t 2 a_{3}\right)$.

Remark 1.4. When the isotropy weights $q, r, p \in\{n, m, k\}$ satisfy $q \neq$ $r(\bmod p)$ only the first case is possible and so, like in the semifree case ([T-W1]), a Theorem of Wall $[\mathbf{W}]$ shows that $M$ (being simply-connected) is diffeomorphic to $S^{2} \times S^{2} \times S^{2}$.

\section{Equivariant cohomology}

Let $E$ be an $S^{1}$-equivariant line bundle over a fixed point $F$. The action of $S^{1}$ on $E$ is conjugate to some circle action on $\mathbf{C}, z \mapsto e^{2 \pi i \beta u} z$ and the first equivariant Chern class is given by $c_{1}^{S^{1}}(E)=\beta u$. Moreover, the normal bundle to $F$ in $M$ decomposes equivariantly into complex line bundles $\nu_{P}=$ 
$L_{1} \oplus \cdots \oplus L_{m}$ on which the circle acts with weights $\beta_{1}, \ldots, \beta_{m}$. Hence, the first two equivariant Chern classes of $\nu_{P}$ are given by

$$
c_{1}^{S^{1}}\left(\nu_{P}\right)=\left(\sum_{i=1}^{m} \beta_{i}\right) u \text { and } c_{2}^{S^{1}}\left(\nu_{P}\right)=\left(\sum_{i, j=1, i \neq j}^{m} \beta_{i} \beta_{j}\right) u^{2},
$$

and the equivariant Euler class by $e_{1}^{S^{1}}\left(\nu_{F}\right)=\left(\prod_{i=1}^{m} \beta_{i}\right) u^{m}$, where $u$ is the generator of $H_{S^{1}}^{2}(F, \mathbf{Z})$. The projection $M \times_{S^{1}} E S^{1} \longrightarrow B S^{1}$, where $E S^{1}$ is a contractible space on which $S^{1}$ acts freely and $B S^{1}=E S^{1} / S^{1}$, induces a push-forward map $p_{*}: H_{S^{1}}^{*}(M, \mathbf{Z}) \longrightarrow H^{*}\left(B S^{1}, \mathbf{Z}\right)$ which is usually denoted by $\int_{M}$ (integration over the fiber), and given by the following localization theorem,

Theorem 2.1. ([B-G-V], $[\mathbf{A}-\mathbf{B}])$ Let $M^{2 n}$ be a compact manifold equipped with an action of $S^{1}$. Let $\alpha \in H_{S^{1}}^{*}(M, \mathbf{Z})$. Then, as elements of $\mathbf{Q}(u)$,

$$
\int_{M} \alpha=\sum_{F \in \mathrm{Fix}_{S^{1}}(M)} \frac{\left.\alpha\right|_{F}}{e_{1}^{S^{1}}\left(\nu_{F}\right)},
$$

where $\nu_{F}$ is the normal bundle to the fixed point $F$ and $e_{1}^{S^{1}}\left(\nu_{F}\right)$ is its equivariant Euler class.

Moreover, Kirwan's injectivity theorem $[\mathbf{K i}]$ relating the equivariant integral cohomology of the manifold $M$ with the equivariant integral cohomology of its fixed point set still holds for circle actions with isolated fixed points (since the cohomology of the fixed point set has no torsion) and states the following:

Theorem 2.2. ([Ki] $]$, [T-W2]) Let $(M, \omega)$ be a compact symplectic manifold equipped with a Hamiltonian circle action with isolated fixed points and let Fix $_{S^{1}}(M)$ be its fixed point set. Then the inclusion map $i: \operatorname{Fix}_{S^{1}}(M) \rightarrow M$ induces an injection $i^{*}: H_{S^{1}}^{*}(M, \mathbf{Z}) \rightarrow H_{S^{1}}^{*}\left(\operatorname{Fix}_{S^{1}}(M), \mathbf{Z}\right)$.

With this theorem one can prove the following proposition (see $[\mathbf{K i}]$, [T-W1], [T-W2] and $[\mathbf{G}]$ for details):

Proposition 2.3. Let $M$ be a symplectic manifold with a Hamiltonian circle action with isolated fixed points. For any $F \in \operatorname{Fix}_{S^{1}}(M)$ of index $2 d$ there is a class $\alpha_{F} \in H_{S^{1}}^{2 d}$ that,

(i) restricted to $F$, is equal to the equivariant Euler class of the negative normal bundle of $F$, that is, $\left.\alpha_{F}\right|_{F}=\left(\prod_{i=1}^{d} \beta_{i}\right) u^{d}$, where $\beta_{1}, \ldots, \beta_{d}$ are the negative isotropy weights of the circle action on the normal bundle of $F$;

(ii) vanishes when restricted to any other fixed point $F^{\prime}$ which cannot be joined to $F$ along a sequence of integral lines of the negative gradient field $-\nabla H$ (where $H$ is the Hamiltonian function of the action). 
Moreover, taken together over all fixed points, these classes are a basis for the cohomology $H_{S^{1}}^{*}(M, \mathbf{Z})$ as a $H^{*}\left(B S^{1}, \mathbf{Z}\right)$-module. We will call them generating classes.

\section{Isolated fixed points on 6-manifolds}

In this section we extend the results of Tolman and Weitsman in [T-W1] to certain non-semifree circle actions with isolated fixed points. For this we first need to consider some facts about isotropy spheres.

3.1. Isotropy spheres. Let $M$ be a compact symplectic six-dimensional manifold equipped with an effective circle action with only isolated fixed points. Let $F \in M$ be one fixed point with at least one isotropy weight on its normal bundle different from \pm 1 . Let $k$ be the absolute value of this weight, and let $M_{k}$ be the connected component of $\operatorname{Fix}_{\mathbf{Z}_{k}}(M)$ containing $F$. If none of the other isotropy weights of $F$ is a multiple of $k$, the tangent space to $M_{k}$ at $F$ is the two-dimensional subspace of $T_{p} M$ on which the circle acts with weight $\pm k$. Consequently, $M_{k}$ is also two-dimensional. Moreover, the circle $S^{1} / \mathbf{Z}_{k}$ acts effectively on this manifold with at least one fixed point $F$. Hence, we have a Hamiltonian action on the compact manifold $M_{k}$ and the image $H\left(M_{k}\right)$ of the corresponding Hamiltonian function is a closed interval. Therefore, there is one additional fixed point on the pre-image of the other endpoint of this interval and so, the local normal forms for circle actions on surfaces, allow us to construct an equivariant symplectomorphism between $M_{k}$ and a sphere with the standard circle action (cf. [K] for details). We conclude that, under the above assumptions, the connected component of $\operatorname{Fix}_{\mathbf{Z}_{k}}(M)$ through $F$ is a sphere containing only one additional fixed point.

If there is one weight which is a multiple of $k$, then the connected component of $\operatorname{Fix}_{\mathbf{Z}_{k}}(M)$ through $F, M_{k}$, is four-dimensional. Nevertheless, there is still an embedded sphere through $F$ which contains only one additional fixed point. In fact, we have a semifree action of $S^{1} / \mathbf{Z}_{k}$ on $M_{k}$ with only isolated fixed points which extends to an action of the multiplicative group $\mathbf{C}^{*}$ on $M_{k}$. The fixed points of this new action are the same as the fixed points of the circle action and the gradient flow on $M_{k}$ is the flow generated by the vector field $-J X$ (where $X$ is the vector field generating the circle action and $J$ is an almost complex structure on $M_{k}$ preserved by the circle action). This gradient flow coincides with the gradient flow of the Hamiltonian function with respect to a compatible metric. We can therefore consider gradient spheres inside $M_{k}$ (defined in $[\mathbf{A}]$ and $[\mathbf{A}-\mathbf{H}]$ ) as the closure of a non-trivial $\mathbf{C}^{*}$-orbit. The poles of these spheres are the limits at times $\infty$ and $-\infty$ of the gradient flow inside this orbit which are, of course, fixed points of the circle action on $M_{k}$. Again, the circle acts on each of these spheres by standard rotation. We conclude then that there is a sphere 
fixed by $\mathbf{Z}_{k}$ through the fixed point $F$ which passes through an additional fixed point in $M$. We will call these spheres isotropy spheres.

3.2. Hamiltonian circle actions. Besides the above considerations on isotropy spheres we will also need the following lemma due to Ahara and Hattori on disc bundles over $S^{2}$ (cf. $[\mathbf{A}-\mathbf{H}]$ for details):

Lemma 3.1. Let the circle act on the two-sphere by rotating it $k$ times while fixing the north and south poles. Let $E \longrightarrow S^{2}$ be a complex line bundle to which the action lifts. The fiber over the north pole is acted upon by $\lambda$ : $z \mapsto \lambda^{m_{N}} z$ and the fibre over the south pole is acted upon by $\lambda: z \mapsto \lambda^{m_{S}} z$. Then, $m_{N}-m_{S}=-e k$, where $e$ is the Euler number of the bundle $E$.

With this lemma we can prove the following theorem:

Theorem 3.2. Let the circle act on a six-dimensional symplectic, compact connected manifold. If all fixed points are isolated and their isotropy weights are always $( \pm n, \pm m, \pm k)$ for fixed integers $n \geq m \geq k \geq 1$, such that $n \neq m+k$, then if $\operatorname{Fix}_{S^{1}}(M) \neq \emptyset$, the action must be Hamiltonian.

Proof. In this proof we will denote by $N_{p},(p=n, m, k)$ the number of fixed points of index 4 with positive isotropy weight equal to $p$, and by $N_{-p}$ the number of fixed points of index 2 and negative isotropy weight equal to $-p$.

If the action is semifree this result is proved by Tolman and Weitsman in [T-W1], so we will only consider here the existence of non-trivial isotropy subgroups $\mathbf{Z}_{k}$. We can assume, without loss of generality, that $\omega$ is rational. Then, if the action is not Hamiltonian, we have that $[\iota(X) \omega] \neq 0$ and so a multiple of $\omega$ admits a generalized moment map $\mu: M \longrightarrow S^{1}$, with $\iota(X) \omega=\mu^{*}(d \theta)$. This map cannot have any local extremum. Consequently, the index of any of its critical points can only be two or four.

Case 1 Let us first consider the case where $n>m=k$. The four possible kinds of fixed points are listed in Figure 1 below: The points of type $(a)$ and $(d)$ cannot be paired to any other fixed point along the $\mathbf{Z}_{n}$-sphere. In fact by Lemma 3.1 , this would imply $2 k \equiv 0(\bmod n)$ (that is $n=2 k$, as $n>k$ ) but, by assumption, $n \neq m+k=2 k$. Therefore, the fixed point set has to be formed uniquely by points of type $(b)$ and $(c)$. Moreover, by Theorem 2.1,

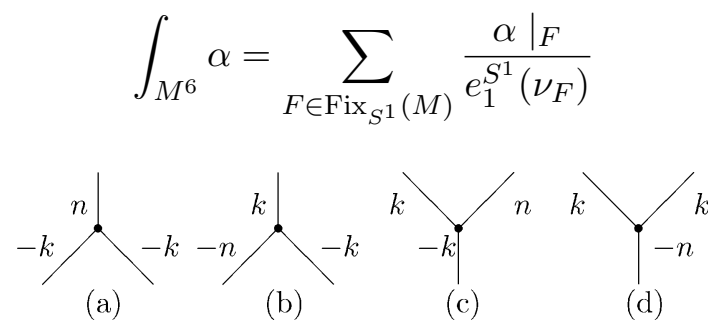

Figure 1. Possible kinds of fixed points. 
for any $\alpha \in H_{S^{1}}^{*}$. If we take $\alpha=c_{1}^{S^{1}}(T M)$ then, for $F$ a fixed point of type $(b), e_{1}^{S^{1}}\left(\nu_{F}\right)=k^{2} n u^{3}$ and $\left.\alpha\right|_{F}=-n u$. Similarly, for $F$ of type $(c)$, $e_{1}^{S^{1}}\left(\nu_{F}\right)=-k^{2} n u^{3}$ and $\left.\alpha\right|_{F}=n u$. Since, by dimensional reasons, $\int_{M^{6}} \alpha=0$, we have

$$
0=\sum_{F \in \operatorname{Fix}_{S^{1}}(M)} \frac{\left.\alpha\right|_{F}}{e_{1}^{S^{1}}\left(\nu_{F}\right)}=-\frac{1}{k^{2} u^{2}}\left(N_{k}+N_{-k}\right)
$$

and so $N_{k}=N_{-k}=0$, contradicting the fact that $\operatorname{Fix}_{S^{1}}(M) \neq \emptyset$. We conclude that there must be at least one point of index zero or six and so the action must be Hamiltonian in this case.

Case 2 Let us now assume that $n \geq m>k=1$. Let $x$ be a fixed point of index 4 , with isotropy weights $(p,-q,-1)$, where $(p, q) \in\{(n, m),(m, n)\}$. For this point to be paired with another fixed point along a $\mathbf{Z}_{p}$-sphere (Figure 2) we need at least one of the following three conditions:

$$
\begin{aligned}
p & =2 \\
q & \equiv-1 \quad(\bmod p) \\
2 q & \equiv 0 \quad(\bmod p) .
\end{aligned}
$$

Let us first consider the case $p \neq 2$ and suppose that $N_{p} \neq 0$. Then, either $q \equiv-1(\bmod p)$ or $2 q \equiv 0(\bmod p)$. If, in addition, $N_{q} \neq 0$, to pair a fixed point $w$ ' with index 4 and positive weight $q$ along the $\mathbf{Z}_{q}$-sphere, one of the following conditions has to be satisfied:

$$
\begin{aligned}
q & =2 \\
p & \equiv-1 \quad(\bmod q) \\
2 p & \equiv 0 \quad(\bmod q) .
\end{aligned}
$$

If $q=2$, (implying that $m=2$ ), then by (2) and (3), we either have $n=p=2,3$ or 4 which are impossible since $p \neq q+1$ and, by Lemma 3.3 below, $p \neq q$ and $p \neq 2 q$.

If $p \equiv-1(\bmod q)$ and $q \equiv-1(\bmod p)$ then $p=-1+s q$ and $q=-1+t p$ for some integers $s, t \geq 2($ as $n \neq m+k=m+1)$ and so, $(s t-1) p=1+s$.

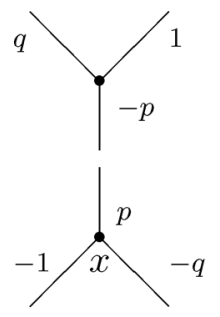

(I)

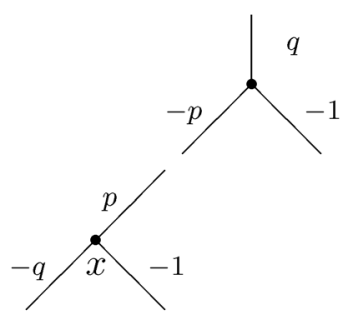

$(I I)$

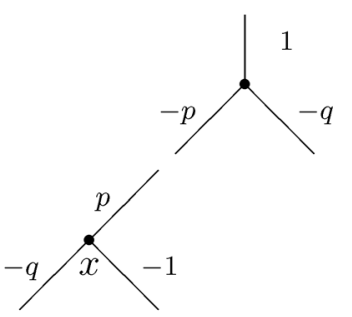

$(I I I)$

Figure 2. Pairings of $x$ along the $\mathbf{Z}_{p}$-sphere. 
As $s t-1 \geq 2 s-1 \geq s+1$ and $p>1$, conditions (5) and (2) cannot be simultaneously satisfied.

If $p \equiv-1(\bmod q)$ and $2 q \equiv 0(\bmod p)$ then $p=-1+s q$ and $2 q=t p$ for integers $s \geq 2$ and $t \geq 1$. Hence $(s t-2) p=2$, which is impossible as st $-2 \geq 1$ and $p>2$ i.e. conditions (5) and (3) cannot be simultaneously satisfied.

Similarly, $2 p \equiv 0(\bmod q)$ and $q \equiv-1(\bmod p)((6)$ and $(2))$ cannot be simultaneously satisfied and so $2 p \equiv 0(\bmod q)$ and $2 q \equiv 0(\bmod p)$ (conditions (6) and (3)) are the only possibly compatible conditions, implying that $2 p=q, 2 q=p$ or $p=q$. However, by Lemma 3.3 below this is impossible and so, either $N_{ \pm p}=0$ or $N_{ \pm q}=0$.

Let us assume that $N_{ \pm p}=0$ (the case where $N_{ \pm q}=0$ is similar). By Theorem 2.1, taking $\alpha=c_{1}^{S^{1}}(T M)$, we have

$0=\sum_{F \in \mathrm{Fix}_{S^{1}}(M)} \frac{\left.\alpha\right|_{F}}{e_{1}^{S^{1}}\left(\nu_{F}\right)}=-\frac{1}{p q u^{2}}\left((p+q-1)\left(N_{1}+N_{-1}\right)+(1+p-q)\left(N_{q}+N_{-q}\right)\right)$.

If $N_{ \pm q} \neq 0$, one of conditions (4), (5) or (6) must hold, that is, either $q=2$, $p=-1+t q(t \geq 2)$ or $2 p=s q$ (with $s \geq 3$ as $q \neq p, 2 p)$. In all cases $(1+p-q)>0$, contradicting the fact that $N_{q} \neq \emptyset$.

If $N_{ \pm q}=0$, then by (7), $N_{ \pm 1}=0$, contradicting the fact that $\operatorname{Fix}_{S^{1}}(M) \neq \emptyset$.

Suppose now that $p=2$ and consider a fixed point $w$ of index 4 and positive weight $q$. To pair $w$ along the $\mathbf{Z}_{q}$-sphere, we would need conditions similar to (1), (2) and (3) now interchanging $p$ with $q$ (that is, $q=1,2,3$ or 4). However, these are all impossible as $q>1, q \neq p+1$ and $q \neq 2,4$ by Lemma 3.3. We conclude then that a point such as $w$ cannot exist and so $N_{q}=0$. Similarly, we can conclude that $N_{-q}=0$ and so, by Theorem 2.1, taking $\alpha=c_{1}^{S^{1}}(T M)$, we have

$$
0=\sum_{F \in \mathrm{Fix}_{S^{1}}(M)} \frac{\left.\alpha\right|_{F}}{e_{1}^{S^{1}\left(\nu_{F}\right)}}=-\frac{1}{2 q u^{2}}\left((q-1)\left(N_{2}+N_{-2}\right)+(1+q)\left(N_{1}+N_{-1}\right)\right),
$$

contradicting the fact that $\operatorname{Fix}_{S^{1}}(M) \neq \emptyset$.

Hence, to finish the proof of this case, we just need to prove Lemma 3.3 which was used above.

Lemma 3.3. If, as above, $(n, m, k)=(t, t, 1)$ or $(2 t, t, 1)$ for some integer $t>1$, then $\operatorname{Fix}_{S^{1}}(M) \neq \emptyset$.

Proof.

1) If $n=m=t$ and the action is not Hamiltonian, the only kinds of fixed points allowed are the ones described in Figure 3. Then, by 


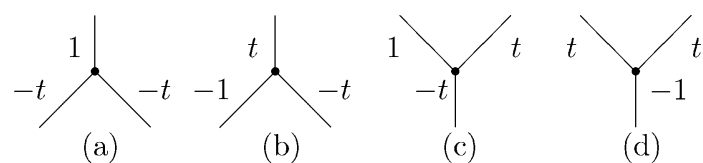

Figure 3. Possible kinds of fixed points.

Theorem 2.1 taking $\alpha=c_{1}^{S^{1}}(T M)$, we have

$$
0=\sum_{F \in \mathrm{Fix}_{S^{1}}(M)} \frac{\left.\alpha\right|_{F}}{e_{1}^{S^{1}}\left(\nu_{F}\right)}=-\frac{1}{t^{2} u^{2}}\left((2 t-1)\left(N_{1}+N_{-1}\right)+\left(N_{t}+N_{-t}\right)\right),
$$

and so $N_{ \pm t}=N_{ \pm 1}=0$, implying that $\operatorname{Fix}_{S^{1}}(M)=\emptyset$.

2) Let us assume now $(n, m)=(2 t, t)$. As all fixed points have to be paired along the $\mathbf{Z}_{j}$-isotropy spheres for $j=n, m$, we need:

$$
\begin{aligned}
N_{1}+N_{t}+N_{-2 t} & =N_{2 t}+N_{-1}+N_{-t} \\
N_{t}+N_{-1}+N_{-2 t} & =N_{2 t}+N_{-t}+N_{1}
\end{aligned}
$$

and so $N_{1}=N_{-1}$ and $N_{t}+N_{-2 t}=N_{2 t}+N_{-t}$. Moreover, since pairings $(I)$ and $(I I)$ in Figure 4 are impossible $(2 \neq 0(\bmod 2 t))$, every fixed point of index 2 and negative weight $-2 t$ has to be paired along the $\mathbf{Z}_{2 t}$-sphere to a fixed point of index 2 and negative weight $-t$, implying that $N_{-2 t} \leq N_{-t}$.

Hence, taking again $\alpha=c_{1}^{S^{1}}(T M)$ in Theorem 2.1, we have

$$
\begin{aligned}
0 & =\sum_{F \in \operatorname{Fix}_{S^{1}}(M)} \frac{\left.\alpha\right|_{F}}{e_{1}^{S^{1}\left(\nu_{F}\right)}} \\
& =\frac{1}{2 t^{2} u^{2}}\left((t-1)\left(N_{2 t}+N_{-2 t}\right)-(t+1)\left(N_{t}+N_{-t}\right)-2(3 t-1) N_{1}\right) \\
& =-\frac{1}{2 t^{2} u^{2}}\left(2 t\left(N_{-t}-N_{-2 t}\right)+2\left(N_{2 t}+N_{-t}\right)+2(3 t-1) N_{1}\right),
\end{aligned}
$$

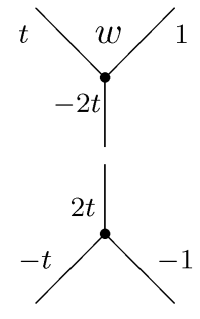

$(I)$

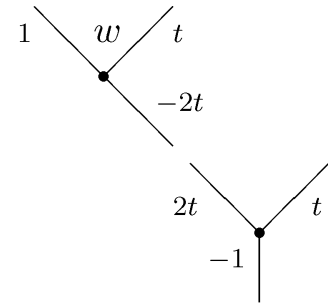

$(I I)$

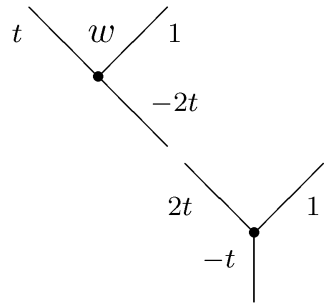

$(I I I)$

Figure 4. Pairings of $w$ along the $\mathbf{Z}_{2 t}$-sphere. 
and so, since $N_{-2 t} \leq N_{-t}$, we have $N_{j}=0$ for $j= \pm 1, \pm t, \pm 2 t$, and again $\operatorname{Fix}_{S^{1}}(M)=\emptyset$.

Case 3 Let us assume now that $n \geq m>k>1$ and consider a fixed point $x$ of index 4 and positive isotropy weight equal to $k$. The possible pairings of $x$ with another fixed point, along the $\mathbf{Z}_{n}$-sphere, are described in Figure 5.

For $(a)$ and $(c)$ to be possible we would need $m \equiv k(\bmod n)$ or $2 k \equiv$ $2 m \equiv 0(\bmod n)$. The first condition is impossible since $n \geq m>k$ and, if the second were true, $k$ would divide both $m$ and $n$ and the action would not be effective. Hence, $(a)$ cannot occur. For $(b)$ to be possible we would need $2 k \equiv 0(\bmod n)$, that is, $2 k=s n$ for some integer $s \geq 1$. However, since $k<n$, we would have $2 k=n$. Finally $(d)$ is always possible. However, if such a pairing of $x$ to a point $w$ occurs, this new point would, in turn, have to be paired to a third fixed point $z$ along the $\mathbf{Z}_{m}$-sphere. These possible pairings are listed in Figure 6.

For one of these to be possible we need one of the following:

$$
\begin{aligned}
n & \equiv-k \quad(\bmod m) \\
m & =2 k \\
2 n & \equiv 0 \quad(\bmod m) .
\end{aligned}
$$

On the other hand, the pairings of $x$ along the $\mathbf{Z}_{m}$-sphere are similar to the ones in Figure 5 (we just have to interchange $n$ with $m$ ). Hence, they can now be of type $(a)$ if $n \equiv k(\bmod m)$, of type $(b)$ if $2 k=m$ and of type $(d)$. If $x$ is paired along the $\mathbf{Z}_{m}$-sphere to a fixed point $w^{\prime}$ of index 2 by a pairing of type $(d)$, this point has in turn to be paired to another fixed point $z$ along the $\mathbf{Z}_{n}$-sphere. The possible pairings are listed in Figure 7 . For one of these to be possible we need one of the following:

$$
\begin{aligned}
n & =2 k \\
2 m & \equiv 0 \quad(\bmod n) .
\end{aligned}
$$

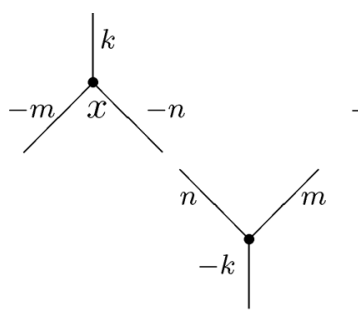

(a)

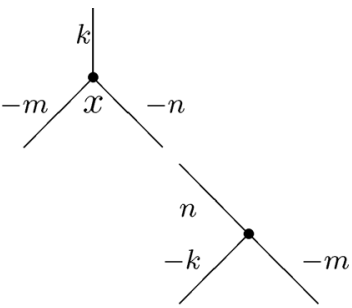

(b)

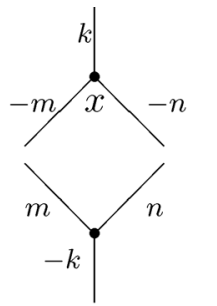

(c)

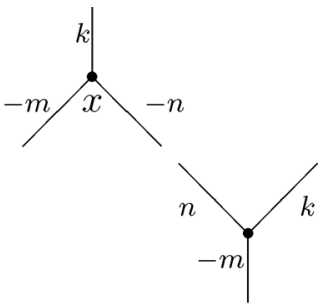

$(d)$

Figure 5. Pairings of $x$ along the $\mathbf{Z}_{n}$-sphere. 


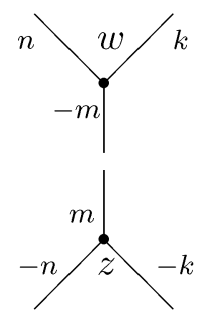

$(I)$

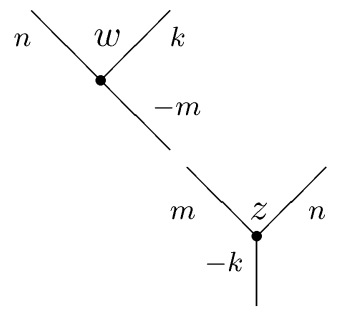

$(I I)$

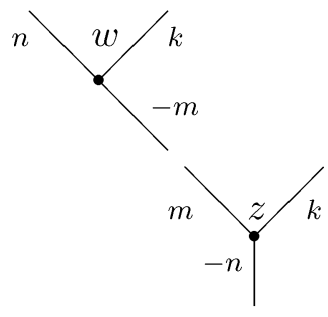

$(I I I)$

Figure 6. Pairings of $w$ along the $\mathbf{Z}_{m}$-sphere.

Note that $(I)$ is impossible since (12) and (13) are not simultaneously satisfied and we cannot have $m \equiv-k(\bmod n)$, as $n \geq m>k>1$ and $n \neq m+k$.

Let us assume for now that $n \neq 2 k$ and $m \neq 2 k$. Then, the only possible pairing of $x$ along the $\mathbf{Z}_{n}$-sphere is of type $(d)$ and for that we need (9) or (11). On the other hand, the only possible pairings of $x$ along the $Z_{m^{-}}$ sphere are of type $(a)$ or $(d)$ and for that we need $n \equiv k(\bmod m)$ or $2 m \equiv 0$ $(\bmod n)$.

If $n \equiv-k(\bmod m)$ and $n \equiv k(\bmod m)$, or if $2 n \equiv 0(\bmod m)$ and $n \equiv k(\bmod m)$, the action would not be effective $(n, m$ and $k$ would have a common divisor).

If $n \equiv-k(\bmod m)$ and $2 m \equiv 0(\bmod n)$, then $2 m=s n$ and $n=-k+t m$ for some integers $2 \geq s \geq 1$ and $t \geq 2$ (as $n \geq m$ ). Hence, $(s t-2) n=2 k$ implying that $s t-2<2$ and so $2 \leq s t<4$ (that is, $s=1$ and $t=2$ or $t=3)$, which is impossible since $n \geq m>k$. Finally, if $2 n \equiv 0(\bmod m)$ and $2 m \equiv 0(\bmod n)$, we would have $2 m=s n$ and $2 n=t m$ for some integers $1 \leq s \leq 2$ and $t \geq 2$. Therefore we would have $4 m=s t m$ and so $s t=4$, implying either $n=2 m$ or $n=m$. We conclude then that one of these conditions must hold and that the pairings of $w$ and $w$ ' along the $\mathbf{Z}_{m}$

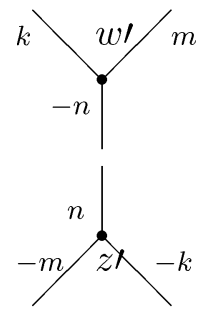

$(I)$

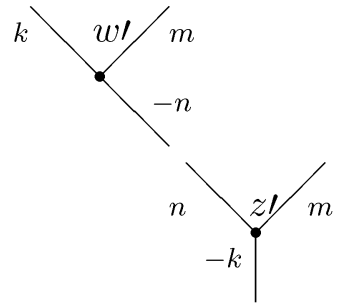

$(I I)$

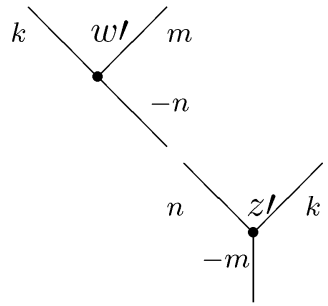

$(I I I)$

Figure 7. Pairings of $w^{\prime}$ along the $\mathbf{Z}_{n}$-sphere. 


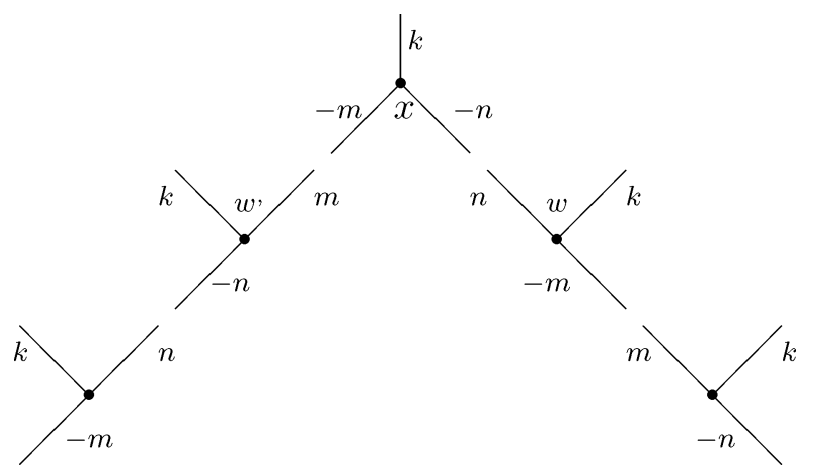

Figure 8. Pairings of $w$ and $w$, along the $\mathbf{Z}_{n}$ and the $\mathbf{Z}_{m}$-spheres.

and the $\mathbf{Z}_{n}$-spheres respectively, are of the type described in Figures 6 and 7 (III) (cf. Figure 8).

Consequently, $N_{k}$ must be equal to zero. Indeed, if there were a fixed point of index 4 with positive weight $k$, then there would be an infinite number of fixed points of index 2 which is impossible. Hence, if $m, n \neq 2 k$, then $N_{k}=0$. Similarly, we can conclude that, under these conditions, $N_{-k}=0$. Take now a fixed point $x^{\prime}$ of index 4 and positive weight $n$. As $N_{-k}=0$, the only possible pairings of $x^{\prime}$ along the $Z_{m}$-sphere to a fixed point $y^{\prime}$ are described in Figure 9. For $(a)$ to be possible we need $n \equiv k(\bmod m)$. However, if that is the case, $y /$ has to be paired along the $\mathbf{Z}_{n}$-sphere by a pairing of type $(I I I)$ in Figure 7 , implying that $2 m \equiv 0(\bmod n)$. Hence, $n=k+s m$ and $2 m=t n$ for some integers $s \geq 2$ and $2 \geq t \geq 1$ and so, $(2-s t) n=2 k$, which is impossible since $s t \geq 2$. Consequently, the pairing of type $(a)$ in Figure 9 is impossible and the existence of a fixed point like $x /$ implies the existence of a fixed point of index 4 and positive weight $m$ (Figure 9(b)) and so $N_{n} \leq N_{m}$.

Interchanging $n$ with $m$ we can also conclude that $N_{m} \leq N_{n}$ and so $N_{m}=N_{n}$. Indeed, if we take a fixed point $x^{\prime \prime}$ of index 4 and positive weight

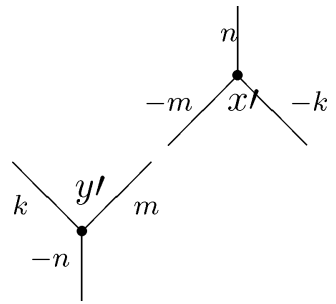

(a)

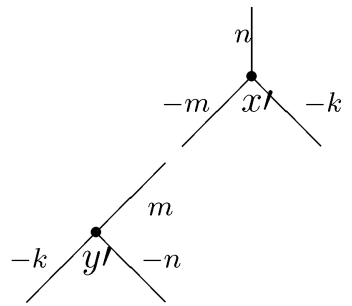

(b)

Figure 9. Pairings of $x^{\prime}$ along the $\mathbf{Z}_{m}$-sphere. 
$m$, then, as $N_{k}=0$, the only possible pairings of $x^{\prime \prime}$ along the $\mathbf{Z}_{n}$-sphere are described in Figure 10. Since we are assuming $m \neq k,(b)$ is in fact the only possible pairing and so $N_{m} \leq N_{n}$. As in addition, all fixed points have to be paired along their isotropy spheres, we have

$$
\begin{aligned}
N_{-m}+N_{n} & =N_{-n}+N_{m} \\
N_{-m}+N_{-n} & =N_{n}+N_{m}
\end{aligned}
$$

implying $N_{n}=N_{-n}=N_{m}=N_{-m}$. By Theorem 2.1, taking $\alpha=c_{1}^{S^{1}}(T M)$, we have

$0=\sum_{F \in \mathrm{Fix}_{S^{1}}(M)} \frac{\left.\alpha\right|_{F}}{e_{1}^{S^{1}}\left(\nu_{F}\right)}=\frac{2}{k m n u^{2}}\left(N_{n}(n-k-m)+N_{m}(m-k-n)\right)=-4 \frac{N_{n}}{m n u^{2}}$,

and so $N_{n}=0$ contradicting the fact that $\operatorname{Fix}_{S^{1}}(M) \neq \emptyset$.

Finally, we will assume that $n=2 k$ (the case where $m=2 k$ is similar). As all fixed points have to be paired along the isotropy spheres, we have:

$$
\begin{aligned}
& N_{-m}+N_{2 k}+N_{k}=N_{-2 k}+N_{-k}+N_{m} \\
& N_{-2 k}+N_{m}+N_{k}=N_{-m}+N_{-k}+N_{2 k} \\
& N_{-k}+N_{m}+N_{2 k}=N_{-m}+N_{-2 k}+N_{k}
\end{aligned}
$$

and so $N_{k}=N_{-k}, N_{m}=N_{-m}$ and $N_{2 k}=N_{-2 k}$.

Moreover, if we take a fixed point $x$ of index 4 and positive weight $m$, the possible pairings along the $Z_{2 k}$-sphere are described in Figure 11. For $(a)$ and (b) to be possible, we would need $2 m \equiv 0(\bmod 2 k)$ and so $k$ would divide both $n$ and $m$ and the action would not be effective. Hence, the existence of a fixed point of index 4 and positive weight $m$ implies the existence of a fixed point of index 2 and negative weight $-k$ (the pairing in $(c)$ is the only one possible) and so $N_{m} \leq N_{-k}=N_{k}$. By Theorem 2.1 using $\alpha=c_{1}^{S^{1}}(T M)$,

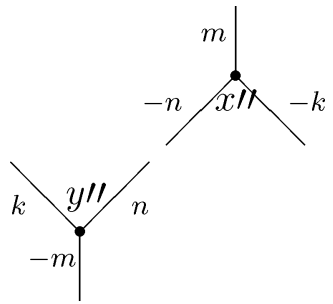

(a)

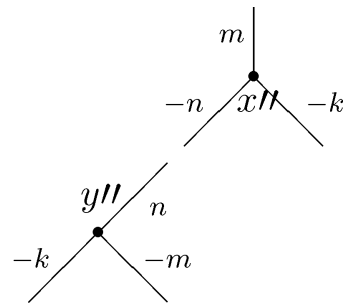

(b)

Figure 10. Pairings of $x / l$ along the $\mathbf{Z}_{n}$-sphere. 


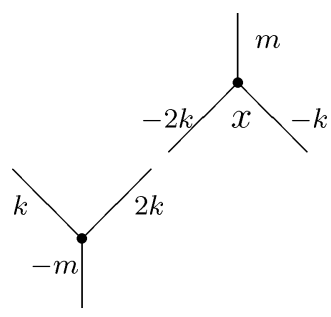

$(a)$

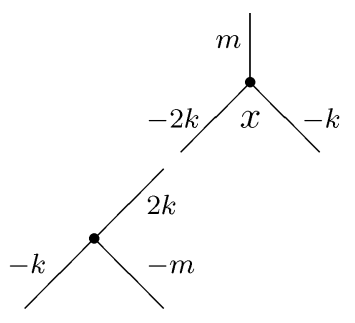

(b)

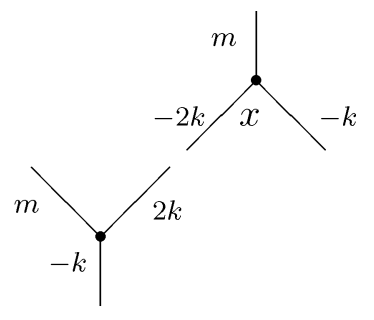

(c)

Figure 11. Pairings of $x$ along the $\mathbf{Z}_{2 k}$-sphere.

we have

$$
\begin{aligned}
0 & =\sum_{F \in \operatorname{Fix}_{S^{1}}(M)} \frac{\left.\alpha\right|_{F}}{e_{1}^{S^{1}\left(\nu_{F}\right)}} \\
& =-\frac{1}{k^{2} m u^{2}}\left(N_{2 k}(m-k)+N_{k}(k+m)+N_{m}(3 k-m)\right) \\
& =-\frac{1}{k^{2} m u^{2}}\left(N_{2 k}(m-k)+k\left(N_{k}+3 N_{m}\right)+m\left(N_{k}-N_{m}\right)\right)
\end{aligned}
$$

and so, as $N_{m} \leq N_{k}$, we have that $N_{k}=N_{2 k}=N_{m}=0$, contradicting the fact that $\operatorname{Fix}_{S^{1}}(M) \neq \emptyset$. We conclude then that the action must be Hamiltonian.

Now that we know that such an action is Hamiltonian, we will show that the number of fixed points is the same as the number of fixed points of the $S^{1}$-space formed by the product of three spheres equipped with a diagonal circle action which rotates each sphere at speed $n, m$ and $k$. Moreover, for every fixed point in $M$ there is a fixed point in $S^{2} \times S^{2} \times S^{2}$ with the same isotropy weights.

Theorem 3.4. Consider a Hamiltonian circle action as in Theorem 3.2. Let $N_{j}$ denote the number of fixed points of index 4 and positive weight $j$. Similarly, let $N_{-j}$ be the number of fixed points of index 2 and negative weight $-j$. Then,

$$
\begin{array}{ll}
N_{ \pm m}=2 N_{ \pm n}=2 & \text { if } n>m=k \\
N_{j}=N_{-j}=1,(j=n, m, k) & \text { if } n>m>k \\
N_{ \pm m}=2 N_{ \pm k}=2 & \text { if } n=m>k
\end{array}
$$

Proof. As the action is Hamiltonian and $M$ is connected, there is only one fixed point of index six and one fixed point of index zero. On the other hand, as all fixed points have to be paired along the non-free isotropy spheres, we 
have, when $n>m>k$,

$$
\begin{aligned}
& N_{n}+N_{-m}+N_{-k}=N_{m}+N_{k}+N_{-n} \\
& N_{m}+N_{-n}+N_{-k}=N_{n}+N_{k}+N_{-m},
\end{aligned}
$$

implying that $N_{k}=N_{-k}$ and $N_{n}+N_{-m}=N_{m}+N_{-n}$. Moreover, by Theorem 2.1, this time taking $\alpha=1$, we have

$$
0=\sum_{F \in \mathrm{Fix}_{S^{1}}(M)} \frac{\left.\alpha\right|_{F}}{e_{1}^{S^{1}}\left(\nu_{F}\right)}=\frac{1}{n m k u^{2}}\left(N_{n}-N_{-n}+N_{m}-N_{-m}+N_{k}-N_{-k}\right),
$$

and so we have $N_{j}=N_{-j}$ for $j=n, m, k$.

If $n=m>k$, we still have $N_{n}+N_{-n}+2 N_{-k}=N_{n}+N_{-n}+2 N_{k}$ and so again $N_{k}=N_{-k}$. Then, by Theorem 2.1, taking $\alpha=1$, we have

$$
0=\sum_{F \in \mathrm{Fix}_{S^{1}}(M)} \frac{\left.\alpha\right|_{F}}{e_{1}^{S^{1}}\left(\nu_{F}\right)}=\frac{1}{n^{2} k u^{2}}\left(N_{n}-N_{-n}+N_{k}-N_{-k}\right),
$$

implying that $N_{j}=N_{-j}$ for $j=n, k$.

If $n>m \geq k$, we have $N_{n}+N_{-m}=N_{m}+N_{-n}$ and so, by Theorem 2.1 with $\alpha=1$, we have

$$
\begin{aligned}
0 & =\sum_{F \in \mathrm{Fix}_{S^{1}}(M)} \frac{\left.\alpha\right|_{F}}{e_{1}^{S^{1}\left(\nu_{F}\right)}}=\frac{1}{n m^{2} u^{2}}\left(N_{n}-N_{-n}+N_{m}-N_{-m}\right) \\
& =\frac{2}{n m^{2} u^{2}}\left(N_{n}-N_{-n}\right),
\end{aligned}
$$

implying that $N_{j}=N_{-j}$ for $j=n, m$. We conclude that, in all cases, we have $N_{j}=N_{-j}$ for $j=n, m, k$.

Consider now a fixed point of index 4 and positive weight $n$. If $n \neq$ $m, 2 k, 2 m$, the only possible pairing of this point along the $\mathbf{Z}_{n}$-sphere is to the fixed point of index 6 and so $N_{n} \leq 1$. Moreover, the only possible pairing of the fixed point of index 6 along the corresponding $\mathbf{Z}_{n}$-sphere is to a fixed point of index 4 and positive weight $n$, and so $N_{n}=1$.

Case 1 First let us consider the case where $n>m=k$. Here $n \neq 2 m, 2 k, m$ and so, as we have seen above, $N_{n}=1$. Moreover, using Theorem 2.1 with $\alpha=c_{1}^{S^{1}}(T M)$, we have

$$
\begin{aligned}
0 & =\sum_{F \in \mathrm{Fix}_{S^{1}}(M)} \frac{\left.\alpha\right|_{F}}{e_{1}^{S^{1}}\left(\nu_{F}\right)} \\
& =\frac{1}{m^{2} n u^{2}}\left((n-2 m)\left(N_{n}+N_{-n}\right)-n\left(N_{m}+N_{-m}\right)+2(n+2 m)\right),
\end{aligned}
$$

and so, as $N_{j}=N_{-j}$ for $j=n, m$ and $N_{n}=1$, we have $n\left(2-N_{m}\right)=0$, implying that $N_{m}=2$ and the result follows. 
Case 2 If $n>m>k$ and $n \neq 2 m, 2 k$, we have again $N_{n}=1$ and $N_{j}=N_{-j}$ for $j=n, m, k$. Hence, by Theorem 2.1 using $\alpha=c_{1}^{S^{1}}(T M)$, we have

$$
\begin{aligned}
0 & =\sum_{F \in \mathrm{Fix}_{S^{1}}(M)} \frac{\left.\alpha\right|_{F}}{e_{1}^{S^{1}}\left(\nu_{F}\right)} \\
& =\frac{2}{n m k u^{2}}\left(2 n+(m-n-k) N_{m}+(k-n-m) N_{k}\right) .
\end{aligned}
$$

Consider now a fixed point of index 4 and positive weight $k$. As $n \neq$ $2 k, m, 2 m$, the only possible way of pairing this fixed point along the $\mathbf{Z}_{n^{-}}$ sphere is to a fixed point of index 2 and negative weight $-m$, implying that $N_{k} \leq N_{m}$. Similarly, the only way of pairing a fixed point of index 4 and positive weight $m$ along the $\mathbf{Z}_{n}$-sphere is to a fixed point of index 2 and negative weight $-k$, and so $N_{m}=N_{k}$. Thus by (16), we have $N_{k}=1$ and the result follows.

Case 3 If $n=2 k>m>k>3$ then, necessarily $2 n \neq 0(\bmod m), n \neq 0$ $(\bmod m), k \neq 0(\bmod m)$ and $3 k \neq 0(\bmod m)$. Hence, the only possible pairing of a fixed point of index 4 and positive weight $m$ along the $\mathbf{Z}_{m}$-sphere is to the fixed point of index 6 and so $N_{m} \leq 1$. Similarly, the only possible pairing of the fixed point of index 6 along the $\mathbf{Z}_{m}$-sphere is to a fixed point of index 4 and positive weight $m$, and so $N_{m}=1$. As, in addition, $N_{j}=N_{-j}$ for every $j$, applying Theorem 2.1 to $\alpha=c_{1}^{S^{1}}(T M)$ gives

$$
\begin{aligned}
0 & =\sum_{F \in \operatorname{Fix}_{S^{1}}(M)} \frac{\left.\alpha\right|_{F}}{e_{1}^{S^{1}\left(\nu_{F}\right)}} \\
& =\frac{1}{k^{2} m u^{2}}\left((k-m) N_{2 k}+(m-3 k) N_{m}-(m+k) N_{k}+3 k+m\right) \\
& =\frac{1}{k^{2} m u^{2}}\left(2 m-(m-k) N_{2 k}-(m+k) N_{k}\right),
\end{aligned}
$$

and so,

$$
2 m=(m+k) N_{k}+(m-k) N_{2 k} .
$$

If $N_{k}$ and $N_{2 k}$ were both different from zero, then $N_{k}+N_{2 k} \geq 2$ and so

$$
k\left(N_{2 k}-N_{k}\right)=m\left(N_{k}+N_{2 k}-2\right) \geq k\left(N_{k}+N_{2 k}-2\right)
$$

which is impossible unless $N_{k}=N_{2 k}=1$. Moreover, as $2 m \neq 0(\bmod 2 k)$ and $m \neq-k(\bmod 2 k)$, the only possible pairings of a fixed point of index 4 and positive weight $2 k$ along the $\mathbf{Z}_{2 k}$-sphere, are either to the fixed point of index 6 or to a fixed point of index 4 and positive weight $k$. Hence, $N_{2 k} \leq N_{k}+1$ and so, if $N_{k}=0$, then necessarily $N_{2 k}=1$ and so by (18), we would have $m=-k$ which is impossible. If, on the other hand, $N_{2 k}=0$ then by (18) we would have $\left(2-N_{k}\right) m=k N_{k}$ and so $N_{k}=1$ and $m=k$ and the 
action would not be effective. We conclude that necessarily $N_{k}=N_{2 k}=1$ and the result follows.

Case 4 If $n=2 k>m>k=2$ then necessarily $n=4$ and $m=3$. As in the preceding case we still have $N_{4} \leq N_{2}+1$. Moreover, as a fixed point of index 4 and positive weight 3 can only be paired along the $\mathbf{Z}_{4}$-isotropy sphere to the fixed point of index 0 or to a fixed point of index 2 and negative weight -2 , we also have $N_{3} \leq N_{2}+1$. On the other hand, by Theorem 2.1 using $\alpha=c_{1}^{S^{1}}(T M)$, we have

$$
0=\sum_{F \in \mathrm{Fix}_{S^{1}}(M)} \frac{\left.\alpha\right|_{F}}{e_{1}^{S^{1}}\left(\nu_{F}\right)}=-\frac{1}{12 u^{2}}\left(N_{4}+3 N_{3}+5 N_{2}-9\right),
$$

implying that $N_{2} \leq 1$. Moreover, as $N_{3} \leq N_{2}+1$ and $N_{4} \leq N_{2}+1$, we have $9 N_{2}-5 \geq 0$ and so $N_{2}=1, N_{4} \leq 2, N_{3} \leq 1$ and $N_{4}+3 N_{3}=4$. Consequently all the $N_{j}$ 's must be greater than zero (therefore equal to 1 ).

Case 5 If $n=2 k>m>k=3$ then necessarily $n=6$ and $m=4$ or $m=5$. If $m=4$, the pairing of a fixed point of index 4 and positive weight 6 along the $\mathbf{Z}_{3}$-isotropy sphere can only be made to a fixed point of index 4 and positive weight 3 , or to a fixed point of index 2 and negative weight -4 , implying that $N_{6} \leq N_{3}+N_{4}$. Moreover, by Theorem 2.1 using $\alpha=c_{1}^{S^{1}}(T M)$, we have

$$
0=\sum_{F \in \operatorname{Fix}_{S^{1}}(M)} \frac{\left.\alpha\right|_{F}}{e_{1}^{S^{1}}\left(\nu_{F}\right)}=-\frac{1}{36 u^{2}}\left(N_{6}+5 N_{4}+7 N_{3}-13\right) .
$$

Then, $N_{3} \leq 1$ and so, as $N_{6} \leq N_{3}+N_{4}$, all the $N_{j}$ 's must be different from zero, implying that they must all be equal to 1 and the result follows.

If $m=5$, the only possible pairing of a fixed point of index 4 and positive weight 6 along the $\mathbf{Z}_{5}$-isotropy sphere is to a fixed point of index 2 and negative weight -3 . Similarly, the only possible pairing of one fixed point of index 2 and negative weight -3 along the $\mathbf{Z}_{5}$-sphere is to a fixed point of index 4 and positive weight 6 , and we conclude that $N_{6}=N_{3}$. On the other hand, by the Theorem 2.1 using $\alpha=c_{1}^{S^{1}}(T M)$, we have

$$
\begin{aligned}
0 & =\sum_{F \in \mathrm{Fix}_{S^{1}}(M)} \frac{\left.\alpha\right|_{F}}{e_{1}^{S^{1}}\left(\nu_{F}\right)} \\
& =-\frac{2}{45 u^{2}}\left(N_{6}+2 N_{5}+4 N_{3}-7\right)=-\frac{2}{45 u^{2}}\left(5 N_{6}+2 N_{5}-7\right),
\end{aligned}
$$

and so $N_{6}=N_{3}=N_{5}=1$.

Case 6 If $n=2 m>m>k$ we have $2 k \neq 0(\bmod 2 m)$ and so, the only possible pairings of a fixed point of index 2 and negative weight $-2 m$ along the $\mathbf{Z}_{2 m}$-isotropy sphere are to a fixed point of index 2 and negative weight $-m$, or to the fixed point of index 0 . Hence, $N_{2 m} \leq N_{m}+1$. 
On the other hand, by Theorem 2.1, using $\alpha=c_{1}^{S^{1}}(T M)$, we have

$$
\begin{aligned}
0 & =\sum_{F \in \mathrm{Fix}_{S^{1}}(M)} \frac{\left.\alpha\right|_{F}}{e_{1}^{S^{1}\left(\nu_{F}\right)}} \\
& =\frac{1}{m^{2} k u^{2}}\left((m-k) N_{2 m}-(m+k) N_{m}-(3 m-k) N_{k}+3 m+k\right)
\end{aligned}
$$

and so, as $N_{2 m} \leq N_{m}+1$, we have $4 m-2 k N_{m}-(3 m-k) N_{k} \geq 0$, implying that $N_{k} \leq 1$ (because $\left.2(3 m-k)>4 m\right)$.

If $N_{k}=0$, the only possible pairing of a fixed point of index 2 and negative weight $-m$ along the $\mathbf{Z}_{2 m}$-isotropy sphere is to a fixed point of index 2, implying that $N_{m} \leq N_{2 m}$ and so $0 \leq N_{2 m}-N_{m} \leq 1$. If $N_{2 m}=N_{m}$, then by $(20)$, we have $N_{m}=\frac{3 m+k}{2 k}$ and so $3 m+k \equiv 0(\bmod 2 k)$, implying that $m=\frac{(2 s-1) k}{3}$ for some integer $s \geq 2$, and then $k=3$ (the action would not be effective otherwise). However, if that was the case, the only possible pairings of fixed points of index 2 and negative weight $-m$ along their $\mathbf{Z}_{2 m^{-}}$ isotropy spheres would be to fixed points of index 2 , and the only possible pairing of fixed points of index 2 and negative weight $-2 m$ along their $\mathbf{Z}_{m^{-}}$ isotropy spheres would be to fixed points of index 2. Hence, this case is impossible since we only have a finite number of fixed points. If, on the other hand, $N_{2 m}=1+N_{m}$, then by (20), we have $N_{m}=\frac{2 m}{k}$ and so $k=2$ and $m$ is odd (again the action would not be effective otherwise). However, if that was the case, again the only possible pairings of fixed points of index 2 and negative weight $-m$ along their $\mathbf{Z}_{2 m}$-isotropy spheres would be to fixed points of index 2 , and the only possible pairings of fixed point of index 2 and negative weight $-2 m$ along their $\mathbf{Z}_{m}$-isotropy spheres would be to a fixed point of index 2 . Hence, this case is also impossible.

We conclude that $N_{k}=1$. Then, by (20), we have

$$
N_{m}=\frac{m-k}{m+k} N_{2 m}+\frac{2 k}{m+k},
$$

and so $N_{m}<N_{2 m}+1$, implying that $0 \leq N_{2 m}-N_{m} \leq 1$. If $N_{2 m}-N_{m}=1$, we have, by $(21)$, that $N_{m}=\frac{m+k}{2 k}$, implying that $m+k \equiv 0(\bmod 2 k)$, that is, $m=(2 s-1) k$ for some integer $s \geq 1$ and then $k=1$ (the action would not be effective otherwise), $N_{m}=\frac{m+1}{2}$ and $N_{2 m}=\frac{m+3}{2}$. However, since $N_{k}=1$, we have three possibilities: if a fixed point of index 2 and negative weight $-2 m$ is connected to the fixed point of index 0 along the $2 m$-sphere and there is a fixed point of index 2 and negative weight $-2 m$ (possibly the same) connected to the fixed point of index 4 and positive weight $k$, along the $m$-sphere, we have $2\left(N_{2 m}-1\right) \leq N_{m}$ (since any other such point will have to be connected to two fixed points of index 2 and negative weight $-m$ ); if a fixed point of index 2 and negative weight $-2 m$ is connected to the fixed point of index 0 along the $2 m$-sphere and no other of these points is connected to the fixed point of index 4 and positive weight $k$, 
then $2\left(N_{2 m}-1\right) \leq N_{m}-1$; if no fixed point of index 2 and negative weight $-2 m$ is connected to the fixed point of index 0 along the $\mathbf{Z}_{2 m}$-isotropy sphere nor to the fixed point of index 4 and positive weight $k$, then $2 N_{2 m} \leq N_{m}$. In all cases we have $2\left(N_{2 m}-1\right) \leq N_{m}$, contradicting the assumption that $N_{2 m}=1+N_{m}$.

Consequently, $N_{k}=1$ and $N_{m}=N_{2 m}$ and so, by (20), we conclude that $N_{k}=N_{2 m}=N_{m}=1$.

Case 7 If $n=m>k>2$, we still have $N_{j}=N_{-j}$ for every $j$. Moreover, as in this case $2 n \neq 0(\bmod k)$ (the action would not be effective otherwise), the only possible pairing of a fixed point of index 4 and positive weight $k$ along the $\mathbf{Z}_{k}$-sphere is to the fixed point of index 6 , implying that $N_{k} \leq 1$. Similarly, the only possible pairing of the fixed point of index 6 along the $\mathbf{Z}_{k}$-sphere is to a fixed point of index 4 and positive weight $k$, implying that $N_{k}=1$. By Theorem 2.1, using $\alpha=c_{1}^{S^{1}}(T M)$, we have

$$
\begin{aligned}
0 & =\sum_{F \in \mathrm{Fix}_{S^{1}}(M)} \frac{\left.\alpha\right|_{F}}{e_{1}^{S^{1}}\left(\nu_{F}\right)} \\
& =\frac{2}{n^{2} k u^{2}}\left(-k N_{n}+(k-2 n) N_{k}+(k+2 n)\right)=\frac{2}{n^{2} u^{2}}\left(2-N_{n}\right),
\end{aligned}
$$

and so $N_{n}=2$ and the result follows.

Case 8 If $n=m>k=1$ and $n \neq 2$ then, by Theorem 2.1, using $\alpha=c_{1}^{S^{1}}(T M)$

$$
0=\sum_{F \in \mathrm{Fix}_{S^{1}}(M)} \frac{\left.\alpha\right|_{F}}{e_{1}^{S^{1}}\left(\nu_{F}\right)}=\frac{2}{n^{2} u^{2}}\left(-N_{n}+(1-2 n) N_{1}+(1+2 n)\right),
$$

and so $N_{1} \leq 1$. Moreover, if $N_{1}=0$, a fixed point of index 4 and positive weight $n$ would have to be paired along the $\mathbf{Z}_{n}$-isotropy sphere to another fixed point of index 4 and positive weight $n$ and this is impossible as $N_{n}$ would either be infinite or zero. We conclude that $N_{1}=1$ and then, by (22), $N_{n}=2$.

Case 9 If $n=m=2>k=1$, then from (22) we still have $N_{1} \leq 1$. If $N_{1}=0$, let $P$ be the fixed point of index 2 with no other fixed point of index 2 on its flow up. Moreover, let $\alpha_{P}$ be a 2-form from Proposition 2.3 associated to $P$ and let $Q$ be the fixed point of index 6 . Then, by Theorem 2.1 we have

$$
\begin{aligned}
0 & =\int_{M} \alpha_{P}=\frac{1}{4 u^{3}}\left(-\left.\alpha_{P}\right|_{P}+\left.\sum_{F \in \mathcal{F}} \alpha_{P}\right|_{F}-\left.\alpha_{P}\right|_{Q}\right) \\
& =\frac{1}{4 u^{3}}\left(2 u+\left.\sum_{F \in \mathcal{F}} \alpha_{P}\right|_{F}-\left.\alpha_{P}\right|_{Q}\right),
\end{aligned}
$$




$$
\begin{aligned}
0 & =\int_{M} c_{1}^{S^{1}}(T M) \cdot \alpha_{P}=\frac{1}{4 u^{3}}\left(-\left.u \alpha_{P}\right|_{P}-\left.u \sum_{F \in \mathcal{F}} \alpha_{P}\right|_{F}+\left.5 u \alpha_{P}\right|_{Q}\right) \\
& =\frac{1}{4 u^{2}}\left(2 u-\left.\sum_{F \in \mathcal{F}} \alpha_{P}\right|_{F}+\left.5 \alpha_{P}\right|_{Q}\right)
\end{aligned}
$$

where $\mathcal{F}$ is the set of fixed points of index 4 in the flow up of $P$, and so $\left.\alpha_{P}\right|_{Q}=-u$ and $\left.\sum_{F \in \mathcal{F}} \alpha_{P}\right|_{F}=-3 u$. On the other hand, using again Theorem 2.1 now with $\alpha_{P}^{2}$, we have

$$
\begin{aligned}
0 & =\int_{M} \alpha_{P}^{2}=\frac{1}{4 u^{3}}\left(-\left(\left.\alpha_{P}\right|_{P}\right)^{2}+\sum_{F \in \mathcal{F}}\left(\left.\alpha_{P}\right|_{F}\right)^{2}-\left(\left.\alpha_{P}\right|_{Q}\right)^{2}\right) \\
& =\frac{1}{4 u^{3}}\left(-4 u^{2}+\sum_{F \in \mathcal{F}}\left(\left.\alpha_{P}\right|_{F}\right)^{2}-u^{2}\right),
\end{aligned}
$$

and so $\sum_{F \in \mathcal{F}}\left(\left.\alpha_{P}\right|_{F}\right)^{2}=5 u^{2}$. Hence, we either have all of the $\left.\alpha_{P}\right|_{F}$ 's equal to zero except two which are equal to $-u$ and $-2 u$, or one of the $\left.\alpha_{P}\right|_{F}$ 's is equal to $u$ while all the other four are equal to $-u$ (note that by $(22) \mathcal{F}$ has at most five points).

Moreover, if we use Theorem 2.1 now with $\alpha_{P}^{3}$, we have

$$
\begin{aligned}
\mathbf{Z} \ni \int_{M} \alpha_{P}^{3} & =\frac{1}{4 u^{3}}\left(-\left(\left.\alpha_{P}\right|_{P}\right)^{3}+\sum_{F \in \mathcal{F}}\left(\left.\alpha_{P}\right|_{F}\right)^{3}-\left(\left.\alpha_{P}\right|_{Q}\right)^{3}\right) \\
& =\frac{1}{4 u^{3}}\left(9 u^{3}+\sum_{F \in \mathcal{F}}\left(\left.\alpha_{P}\right|_{F}\right)^{3}\right)
\end{aligned}
$$

and so $\sum_{F \in \mathcal{F}}\left(\left.\alpha_{P}\right|_{F}\right)^{3}+u^{3} \in 4 u^{3} \cdot \mathbf{Z}$. Consequently, the second hypothesis above cannot hold and we are left with the first: one of the $\left.\alpha_{P}\right|_{F}$ 's is equal to $-2 u$, another one is equal to $-u$ and all the others are equal to zero. Let $P_{1}$ be the fixed point where $\left.\alpha_{P}\right|_{P_{1}}=-2 u$, let $P_{2}$ be the one where $\left.\alpha_{P}\right|_{P_{2}}=-u$ and let $\alpha_{P_{1}}$ and $\alpha_{P_{1}}$ be 2 -forms from Proposition 2.3 associated to $P_{1}$ and $P_{2}$ respectively. Let us assume first that there is at least one fixed point $P_{3}$ of index 4 different from $P_{2}$ on the flow up of $P_{1}$. If $P_{3}$ were directly connected to $Q$ (the fixed point of index 6) then, using Theorem 2.1 with $\alpha_{P_{3}}$ (a 2-form from Proposition 2.3 associated to $P_{3}$ ), we would have

$$
0=\frac{1}{4 u^{3}}\left(\left.\alpha_{P_{3}}\right|_{P_{3}}-\left.\alpha_{P_{3}}\right|_{Q}\right)=\frac{1}{4 u^{3}}\left(2 u^{2}-\left.\alpha_{P_{3}}\right|_{Q}\right),
$$

implying that $\left.\alpha_{P_{3}}\right|_{Q}=\left.\alpha_{P_{3}}\right|_{P_{3}}=2 u^{2}$. However, taking again Theorem 2.1, now with $\alpha_{P} \cdot \alpha_{P_{3}}$, we would have $\frac{\left.\left.\alpha_{P}\right|_{Q} \cdot \alpha_{P_{3}}\right|_{Q}}{4 u^{3}}=\frac{1}{2} \in \mathbf{Z}$ which is impossible. 
Moreover, if $P_{1}$ were directly connected to $Q$, we would have,

$$
\mathbf{Z} \ni \int_{M} \alpha_{P} \cdot \alpha_{P_{1}}=\frac{1}{4 u^{3}}\left(-\left.2 u \alpha_{P_{1}}\right|_{P_{1}}+\left.u \alpha_{P_{1}}\right|_{Q}\right)=-\frac{1}{2}
$$

which is impossible. We conclude then that $P_{2}$ is in the flow up of $P_{1}$ and that it is directly connected to $Q$. (Note that, in this case, we have

$$
\left.\int_{M} \alpha_{P} \cdot \alpha_{P_{2}}=\frac{1}{4 u^{3}}\left(-\left.u \alpha_{P_{2}}\right|_{P_{2}}+\left.u \alpha_{P_{2}}\right|_{Q}\right)=\frac{1}{4 u^{3}}\left(-2 u^{3}+2 u^{3}\right)=0 \in \mathbf{Z}\right) .
$$

Let now $P_{3}$ denote the fixed point different from $P_{2}$ (if it exists) in the flow up of $P_{1}$ and such that $P_{2}$ and $Q$ are the only fixed points on its flow up. Then, using Theorem 2.1, we have

$0=\int_{M} \alpha_{P_{3}}=\frac{1}{4 u^{3}}\left(\left.\alpha_{P_{3}}\right|_{P_{3}}+\left.\alpha_{P_{3}}\right|_{P_{2}}-\left.\alpha_{P_{3}}\right|_{Q}\right)=\frac{1}{4 u^{3}}\left(2 u^{2}+\left.\alpha_{P_{3}}\right|_{P_{2}}-\left.\alpha_{P_{3}}\right|_{Q}\right)$ and so, since $\left.\alpha_{P}\right|_{P_{3}}=0$,

$\mathbf{Z} \ni \int_{M} \alpha_{P} \cdot \alpha_{P_{3}}=\frac{1}{4 u^{3}}\left(-\left.u \alpha_{P_{3}}\right|_{P_{2}}+\left.u \alpha_{P_{3}}\right|_{Q}\right)=\frac{1}{4 u^{2}}\left(-\left.\alpha_{P_{3}}\right|_{P_{2}}+\left.\alpha_{P_{3}}\right|_{Q}\right)=\frac{1}{2}$ which is impossible.

We conclude that there is no fixed point on the flow up of $P_{1}$ other than $P_{2}$. Thus, using Theorem 2.1 with $\alpha_{P_{1}}$, we have

$$
0=\frac{1}{4 u^{3}}\left(\left.\alpha_{P_{1}}\right|_{P_{1}}+\left.\alpha_{P_{1}}\right|_{P_{2}}-\left.\alpha_{P_{1}}\right|_{Q}\right)=\frac{1}{4 u^{3}}\left(2 u^{2}+\left.\alpha_{P_{1}}\right|_{P_{2}}-\left.\alpha_{P_{1}}\right|_{Q}\right),
$$

and so, using $\alpha_{P} \cdot \alpha_{P_{1}}$, we have

$$
\mathbf{Z} \ni \int_{M} \alpha_{P} \cdot \alpha_{P_{1}}=\frac{1}{4 u^{3}}\left(-4 u^{3}-\left.u \alpha_{P_{1}}\right|_{P_{2}}+\left.u \alpha_{P_{1}}\right|_{Q}\right)=-\frac{1}{2}
$$

which is impossible. We conclude then that this case is also impossible and so $N_{1}=1$, implying $N_{2}=2$, and the result follows.

Case 10 If $n=m>k=2$, we have, by Theorem 2.1, using $\alpha=c_{1}^{S^{1}}(T M)$, $0=\frac{2}{2 n^{2} u^{2}}\left(-2 N_{n}+(2-2 n) N_{2}+(2+2 n)\right)=\frac{2}{n^{2} u^{2}}\left(-N_{n}+(1-n) N_{2}+(1+n)\right)$, implying that $N_{n}+(n-1) N_{2}=n+1$. As $n \geq 3, N_{2}$ must be smaller than 3. If $N_{2}=0$, then $N_{n}=n+1$. However, if that was the case, the fixed points of index 4 and positive weight $n$ could only be paired along their "negative" $\mathbf{Z}_{n}$-isotropy spheres, to other fixed points of index 4 and positive weight $n$ (note that $n \neq 4$ as the action would not be effective otherwise). As the number of fixed points is finite, $N_{n}$ would also have to be zero which is impossible. If $N_{2}=2$, then $n$ must be equal to 3 and $N_{n}=0$. However, in this case, the fixed points of index 4 and positive weight 2 , can only be paired along the $\mathbf{Z}_{n}$-isotropy spheres to the fixed point of index 0 which makes this case impossible. We conclude that $N_{2}=1$ and, consequently, $N_{n}=2$. 


\section{Constructing an example}

An obvious example of an $S^{1}$-action on a compact symplectic connected 6dimensional manifold with isolated fixed points satisfying the condition that on their normal bundles, the isotropy weights are always $n \geq m \geq k$, is a diagonal action on the product of three spheres which rotates each sphere at speed $n, m$ and $k$. We will now construct another example which may occur when the isotropy weights satisfy $q \cong r(\bmod p)$ for some $p, q, r \in\{n, m, k\}$, $(p \neq q \neq r)$.

Let us assume that $q=r+e p$ for some integer $e$. Decompose the sphere $S^{2}$ in two hemispheres $S^{2}=S_{+}^{2} \cup S_{-}^{2}$, where $S_{+}^{2}=\{z \in \mathbf{C}:|z| \leq 1\}$ and $S_{-}^{2}=\{z \in \mathbf{C} \cup \infty:|z| \geq\} \cong\{z \in \mathbf{C}:|z| \leq 1\}$. Consider $S^{2} \times S^{2} \times S_{-}^{2}$ with the circle action given by

$$
\lambda \cdot\left(\left[z_{0}: z_{1}\right],\left[u_{0}: u_{1}\right], v\right)=\left(\left[\lambda^{q} z_{0}: z_{1}\right],\left[\lambda^{r} u_{0}: u_{1}\right], \lambda^{p} v\right)
$$

and $S^{2} \times S^{2} \times S_{+}^{2}$ with the circle action

$$
\lambda \cdot\left(\left[z_{0}: z_{1}\right],\left[u_{0}: u_{1}\right], w\right)=\left(\left[\lambda^{r} z_{0}: z_{1}\right],\left[\lambda^{q} u_{0}: u_{1}\right], \lambda^{-p} w\right),
$$

where $w=1 / v$. Then construct the $S^{2} \times S^{2}$-bundle, $E$, over $S^{2}$, gluing $S^{2} \times S^{2} \times S_{-}^{2}$ and $S^{2} \times S^{2} \times S_{+}^{2}$ along their common boundary, $S^{2} \times S^{2} \times S^{1}$, by the gluing map

$$
\varphi\left(\left[z_{0}: z_{1}\right],\left[u_{0}: u_{1}\right], v\right)=\left(\left[v^{-e} z_{0}: z_{1}\right],\left[v^{e} u_{0}: u_{1}\right], w\right) .
$$

Note that $E$ is equipped with a global circle action. Indeed,

$$
\begin{aligned}
\varphi & \left(\lambda \cdot\left(\left[z_{0}: z_{1}\right],\left[u_{0}: u_{1}\right], v\right)\right)=\varphi\left(\left[\lambda^{q} z_{0}: z_{1}\right],\left[\lambda^{r} u_{0}: u_{1}\right], \lambda^{p} v\right) \\
& =\left(\left[\lambda^{q-e p} v^{-e} z_{0}: z_{1}\right],\left[\lambda^{r+e p} v^{e} u_{0}: u_{1}\right], \lambda^{-p} w\right) \\
& =\left(\left[\lambda^{r} v^{-e} z_{0}: z_{1}\right],\left[\lambda^{q} v^{e} u_{0}: u_{1}\right], \lambda^{-p} w\right) \\
& =\lambda \cdot\left(\left[v^{-e} z_{0}: z_{1}\right],\left[v^{e} u_{0}: u_{1}\right], w\right)=\lambda \cdot \varphi\left(\left[z_{0}: z_{1}\right],\left[u_{0}: u_{1}\right], v\right) .
\end{aligned}
$$

We will see in the next section that, when the isotropy weights are pairwise relatively prime, all $S^{1}$-spaces satisfying the above condition on the isotropy weights have the same cohomology as one of these two examples.

\section{Cohomology}

Now that we have shown that our circle actions must have the same fixed point data, (that is, the number of fixed points and their isotropy weights) as the examples in Section 4, we prove that, when the isotropy weights are pairwise relatively prime, they even have the same equivariant cohomology and Chern classes of the above examples, and that, if in addition all the isotropy weights $q, r, p \in\{n, m, k\}$ satisfy $q \neq r(\bmod p)$, the manifold has to be diffeomorphic to the product of three spheres.

Theorem 5.1. Let $(M, \omega)$ be a compact, connected, symplectic 6-manifold equipped with a Hamiltonian circle action with isolated fixed points. Let all 
fixed points satisfy the condition that, on their normal bundles, the isotropy weights are always $n>m>k>2$ pairwise relatively prime, and $n \neq m+k$. Then, there either exists a map from $\operatorname{Fix}_{S^{1}}(M)$ to $\operatorname{Fix}_{S^{1}}\left(\left(S^{2}\right)^{3}\right)$ (the fixed point set of a diagonal circle action on the product of three spheres rotating each one at speeds $n, m, k$ ) or a map from $\operatorname{Fix}_{S^{1}}(M)$ to $\operatorname{Fix}_{S^{1}}(E)$ (the fixed point set of the $S^{1}$-space $E$ described in Section 4) which respectively identifies the restrictions $i^{*}$ and $j^{*}$ or $i^{*}$ and $\tilde{j}^{*}$, of the equivariant cohomology classes to the fixed point sets, where

$$
\begin{aligned}
i^{*}: & H_{S^{1}}^{*}(M, \mathbf{Z}) \longrightarrow H_{S^{1}}^{*}\left(\operatorname{Fix}_{S^{1}}(M), \mathbf{Z}\right) \\
j^{*}: & H_{S^{1}}^{*}\left(\left(S^{2}\right)^{3}, \mathbf{Z}\right) \longrightarrow H_{S^{1}}^{*}\left(\operatorname{Fix}_{S^{1}}\left(\left(S^{2}\right)^{3}\right), \mathbf{Z}\right) \\
\tilde{j}^{*}: & H_{S^{1}}^{*}(E, \mathbf{Z}) \longrightarrow H_{S^{1}}^{*}\left(\operatorname{Fix}_{S^{1}}(E), \mathbf{Z}\right)
\end{aligned}
$$

are induced by the inclusions $i: \operatorname{Fix}_{S^{1}}(M) \rightarrow M, j: \operatorname{Fix}_{S^{1}}\left(\left(S^{2}\right)^{3}\right) \rightarrow\left(S^{2}\right)^{3}$ and $\tilde{j}: \operatorname{Fix}_{S^{1}}(E) \rightarrow E$. Moreover, this map sends the images of equivariant Chern classes of $M$ to those of $S^{2} \times S^{2} \times S^{2}$ or $E$.

Proof. Since the isotropy weights are pairwise relatively prime and greater than 2 , the fixed point of index 0 can only be paired to fixed points of index 2. Indeed, if it were paired along a $Z_{p}$-sphere to a fixed point of index 4 with positive weight $q$ and negative weights $-p,-r(p, q, r \in\{n, m, k\}$ and $p \neq q \neq r$ ) then we would need $2 r \equiv 0 \bmod p$ which is impossible by assumption. By a similar argument, we can see that it cannot be paired to the fixed point of index 6 and that this last point can only be paired to fixed points of index 4 . Let us now see how we can pair the remaining fixed points (cf. Figure 12). We can divide all possibilities in four different cases:

I. All remaining pairings of fixed points are like the ones in $S^{2} \times S^{2} \times S^{2}$ (Figure 12.I);

II. All remaining pairings are like the ones in the product of spheres except the pairings along the isotropy spheres of one order (Figure 12.II);

III. Only the pairings along the isotropy spheres of one order are like the ones in the product of spheres (Figure 12.III);

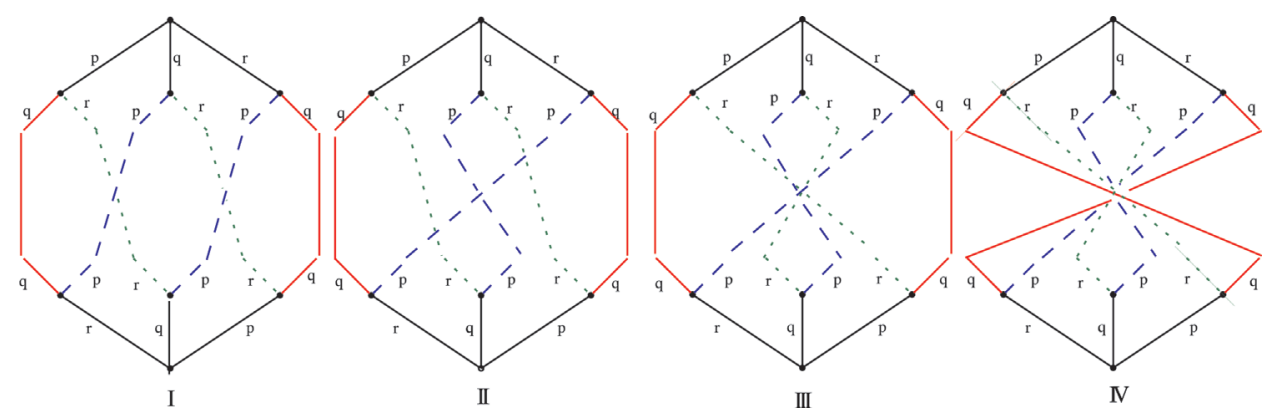

Figure 12. Possible pairings of fixed points. 
IV. None of the remaining pairings is like the ones in $S^{2} \times S^{2} \times S^{2}$ (Figure $12 . \mathrm{IV})$.

We will use Proposition 2.3 to prove this theorem. Indeed, we will use it to show that only the first two cases are possible and to show that the behavior of the corresponding generating equivariant cohomology classes is the same as their counterparts in $S^{2} \times S^{2} \times S^{2}$ or in $E$. Let us first label the fixed points of index 4 and positive weights $p, q$ and $r(p, q, r \in\{n, m, k\})$ respectively by $a, b, c$, the fixed point of index 6 by $d$, and consider generating equivariant 2-classes $\alpha, \beta, \gamma$ corresponding to the fixed points of index 2 which have negative weights $-r,-q,-p$ respectively. Then, we obtain the following sets of equations for the four possible cases:
I.
(i) $r+\left.\alpha\right|_{a}+\left.\alpha\right|_{b}-\left.\alpha\right|_{d}=0$
(iv) $r^{2}-\left.\alpha^{2}\right|_{a}-\left.\alpha^{2}\right|_{b}+\left.\alpha^{2}\right|_{d}=0$
(vii) $\left.\left.\alpha\right|_{a} \beta\right|_{a}=\left.\left.\alpha\right|_{d} \beta\right|_{d}$
(ii) $q+\left.\beta\right|_{a}+\left.\beta\right|_{c}-\left.\beta\right|_{d}=0$
(v) $q^{2}-\left.\beta^{2}\right|_{a}-\left.\beta^{2}\right|_{c}+\left.\beta^{2}\right|_{d}=0$
(viii) $\left.\left.\alpha\right|_{b} \gamma\right|_{b}=\left.\left.\alpha\right|_{d} \gamma\right|_{d}$
(iii) $p+\left.\gamma\right|_{b}+\left.\gamma\right|_{c}-\left.\gamma\right|_{d}=0$
(vi) $p^{2}-\left.\gamma^{2}\right|_{b}-\left.\gamma^{2}\right|_{c}+\left.\gamma^{2}\right|_{d}=0$
(ix) $\left.\left.\beta\right|_{c} \gamma\right|_{c}=\left.\left.\beta\right|_{d} \gamma\right|_{d}$

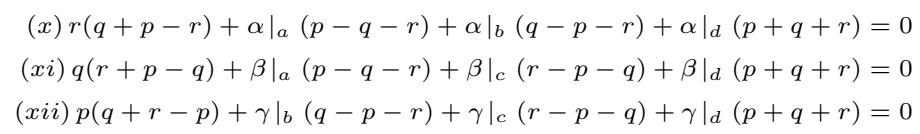
II.
(i) $r+\left.\alpha\right|_{a}+\left.\alpha\right|_{c}-\left.\alpha\right|_{d}=0 \quad$ (iv) $r^{2}-\left.\alpha^{2}\right|_{a}-\left.\alpha^{2}\right|_{c}+\left.\alpha^{2}\right|_{d}=0 \quad$ (vii) $\left.\left.\alpha\right|_{a} \beta\right|_{a}=\left.\left.\alpha\right|_{d} \beta\right|_{d}$
(ii) $q+\left.\beta\right|_{a}+\left.\beta\right|_{b}-\left.\beta\right|_{d}=0$
(v) $q^{2}-\left.\beta^{2}\right|_{a}-\left.\beta^{2}\right|_{b}+\left.\beta^{2}\right|_{d}=0$
(viii) $\left.\left.\alpha\right|_{c} \gamma\right|_{c}=\left.\left.\alpha\right|_{d} \gamma\right|_{d}$
(iii) $p+\left.\gamma\right|_{b}+\left.\gamma\right|_{c}-\left.\gamma\right|_{d}=0$
(vi) $p^{2}-\left.\gamma^{2}\right|_{b}-\left.\gamma^{2}\right|_{c}+\left.\gamma^{2}\right|_{d}=0$
(ix) $\left.\left.\beta\right|_{b} \gamma\right|_{b}=\left.\left.\beta\right|_{d} \gamma\right|_{d}$

(x) $r(q+p-r)+\left.\alpha\right|_{a}(p-q-r)+\left.\alpha\right|_{c}(r-p-q)+\left.\alpha\right|_{d}(p+q+r)=0$
$(x i) q(r+p-q)+\left.\beta\right|_{a}(p-q-r)+\left.\beta\right|_{b}(q-p-r)+\left.\beta\right|_{d}(p+q+r)=0$
(xii) $p(q+r-p)+\left.\gamma\right|_{b}(q-p-r)+\left.\gamma\right|_{c}(r-p-q)+\left.\gamma\right|_{d}(p+q+r)=0$

III.
(i) $r+\left.\alpha\right|_{a}+\left.\alpha\right|_{c}-\left.\alpha\right|_{d}=0$
(iv) $r^{2}-\left.\alpha^{2}\right|_{a}-\left.\alpha^{2}\right|_{c}+\left.\alpha^{2}\right|_{d}=0$
(vii) $\left.\left.\alpha\right|_{d} \beta\right|_{d}=0$
(ii) $q+\left.\beta\right|_{b}-\left.\beta\right|_{d}=0$
(v) $q^{2}-\left.\beta^{2}\right|_{b}+\left.\beta^{2}\right|_{d}=0$
(iii) $p+\left.\gamma\right|_{a}+\left.\gamma\right|_{c}-\left.\gamma\right|_{d}=0$
(vi) $p^{2}-\left.\gamma^{2}\right|_{a}-\left.\gamma^{2}\right|_{c}+\left.\gamma^{2}\right|_{d}=0$
(ix) $\left.\left.\beta\right|_{d} \gamma\right|_{d}=0$
(x) $r(q+p-r)+\left.\alpha\right|_{a}(p-q-r)+\left.\alpha\right|_{c}(r-p-q)+\left.\alpha\right|_{d}(p+q+r)=0$
(xi) $q(r+p-q)+\left.\beta\right|_{b}(q-p-r)+\left.\beta\right|_{d}(p+q+r)=0$
(xii) $p(q+r-p)+\left.\gamma\right|_{a}(p-q-r)+\left.\gamma\right|_{c}(r-p-q)+\left.\gamma\right|_{d}(p+q+r)=0$

(viii) $\left.\left.\alpha\right|_{a} \gamma\right|_{a}+\left.\left.\alpha\right|_{c} \gamma\right|_{c}=\left.\left.\alpha\right|_{d} \gamma\right|_{d}$
IV.
(i) $r+\left.\alpha\right|_{c}-\left.\alpha\right|_{d}=0$
(iv) $r^{2}-\left.\alpha^{2}\right|_{c}+\left.\alpha^{2}\right|_{d}=0$
(ii) $q+\left.\beta\right|_{b}-\left.\beta\right|_{d}=0$
(v) $q^{2}-\left.\beta^{2}\right|_{b}+\left.\beta^{2}\right|_{d}=0$
(iii) $p+\left.\gamma\right|_{a}-\left.\gamma\right|_{d}=0$
(vi) $p^{2}-\left.\gamma^{2}\right|_{a}+\left.\gamma^{2}\right|_{d}=0$

\begin{abstract}
(x) $r(q+p-r)+\left.\alpha\right|_{c}(r-p-q)+\left.\alpha\right|_{d}(p+q+r)=0$
(xi) $q(r+p-q)+\left.\beta\right|_{b}(q-p-r)+\left.\beta\right|_{d}(p+q+r)=0$
\end{abstract}
(xii) $p(q+r-p)+\left.\gamma\right|_{a}(p-q-r)+\left.\gamma\right|_{d}(p+q+r)=0$

(vii) $\left.\left.\alpha\right|_{d} \beta\right|_{d}=0$

(viii) $\left.\left.\alpha\right|_{d} \gamma\right|_{d}=0$

(ix) $\left.\left.\beta\right|_{d} \gamma\right|_{d}=0$ 
corresponding to the equations
(i) $0=\int_{M} \alpha$;
(ii) $0=\int_{M} \beta$;
(iii) $0=\int_{M} \gamma$;
(iv) $0=\int \alpha^{2}$;
(v) $0=\int_{M} \beta^{2}$;
(vi) $0=\int_{M} \gamma^{2}$;
(vii) $0=\int_{M} \alpha \cdot \beta$;
(viii) $0=\int_{M} \alpha \cdot \gamma$;
(ix) $0=\int_{M} \beta \cdot \gamma$;
$(x) 0=\int_{M} c_{1}^{S^{1}}(T M) \cdot \alpha ;$
(xi) $0=\int_{M} c_{1}^{S^{1}}(T M) \cdot \beta$;
(xii) $0=\int_{M} c_{1}^{S^{1}}(T M) \cdot \gamma$.

Let us begin with Cases III and IV. Here, we consider equations ( $i i)$ and $(v)$ and get $\left.\beta\right|_{d}=0$ and $\left.\beta\right|_{b}=-q$, contradicting (xi). Consequently, the only possible cases are the first two.

In case I, equations $(i)$ through (xii) completely determine the values of the non-zero restrictions of $\alpha, \beta$ and $\gamma$ :

$$
\begin{aligned}
& \left.\alpha\right|_{a}=\left.\alpha\right|_{b}=\left.\alpha\right|_{d}=-r u ;\left.\quad \beta\right|_{a}=\left.\beta\right|_{c}=\left.\beta\right|_{d}=-q u ; \\
& \left.\gamma\right|_{b}=\left.\gamma\right|_{c}=\left.\gamma\right|_{d}=-p u .
\end{aligned}
$$

Moreover, if $\bar{\alpha}, \bar{\beta}, \bar{\gamma} \in H_{S^{1}}^{2}(M, \mathbf{Z})$ are generating 4-classes (defined in Proposition 2.3) associated to the fixed points of index 4 and positive weight $p, q, r$ respectively, we get:

$$
\begin{array}{ll}
0 & =\int_{M} \bar{\alpha}=\frac{q r u^{2}-\left.\bar{\alpha}\right|_{d}}{p q r u^{3}} ; \quad 0=\int_{M} \bar{\beta}=\frac{p r u^{2}-\left.\bar{\beta}\right|_{d}}{p q r u^{3}} ; \\
0 & =\int_{M} \bar{\gamma}=\frac{p q u^{2}-\left.\bar{\gamma}\right|_{d}}{p q r u^{3}},
\end{array}
$$

implying that the non-zero restrictions of $\bar{\alpha}, \bar{\beta}, \bar{\gamma}$ to the fixed points are:

$$
\left.\bar{\alpha}\right|_{a}=\left.\bar{\alpha}\right|_{d}=q r u^{2} ;\left.\quad \bar{\beta}\right|_{b}=\left.\bar{\beta}\right|_{d}=p r u^{2} ;\left.\quad \bar{\gamma}\right|_{c}=\left.\bar{\gamma}\right|_{d}=p q u^{2} .
$$

As these classes form a basis for the cohomology $H_{S^{1}}^{*}(M, \mathbf{Z})$, and equations $(i)$ through (xii) and (23) also have to be satisfied by the generating classes of $H_{S^{1}}^{*}\left(\left(S^{2}\right)^{3}, \mathbf{Z}\right)$, the result follows. Moreover, the restrictions of the equivariant Chern classes of the two spaces are also the same since the isotropy weights are the same.

For case II. to be possible we need $q \equiv r(\bmod p)$ in order to have the corresponding pairings along $\mathbf{Z}_{p}$-spheres. If this is the case, equations $(i)$ through (xii) above, completely determine the values of the non-zero restrictions of $\alpha, \beta$ and $\gamma$ :

$$
\begin{aligned}
& \left.\alpha\right|_{a}=-r u,\left.\alpha\right|_{c}=\left.\alpha\right|_{d}=-q u ;\left.\quad \beta\right|_{a}=-q u,\left.\beta\right|_{b}=\left.\beta\right|_{d}=-r u ; \\
& \left.\gamma\right|_{b}=\left.\gamma\right|_{c}=\left.\gamma\right|_{d}=-p u .
\end{aligned}
$$


Moreover, like in Case $I$, if $\bar{\alpha}, \bar{\beta}, \bar{\gamma} \in H_{S^{1}}^{2}(M, \mathbf{Z})$ are generating 4-classes associated to the fixed points of index 4 and positive weight $p, q, r$ respectively, their non-zero restrictions to the fixed points are:

$$
\left.\bar{\alpha}\right|_{a}=\left.\bar{\alpha}\right|_{d}=q r u^{2} ;\left.\quad \bar{\beta}\right|_{b}=\left.\bar{\beta}\right|_{d}=p r u^{2} ;\left.\quad \bar{\gamma}\right|_{c}=\left.\bar{\gamma}\right|_{d}=p q u^{2} .
$$

Again, as these classes form a basis for the cohomology $H_{S^{1}}^{*}(M, \mathbf{Z})$, and equations ( $i$ ) through (xii) and (23) also have to be satisfied by the generating classes of $H_{S^{1}}^{*}(E, \mathbf{Z})$ (note that $q \equiv r(\bmod p)$ ), the result follows. Moreover, the restrictions of the equivariant Chern classes of the two spaces are also the same since the isotropy weights are the same.

Remark 5.2. To prove the above result we didn't really need the isotropy weights to be pairwise relatively prime. Indeed, it still holds if $2 q \neq 0$ $(\bmod r)$ for every $q, r \in\{n, m, k\}$. However, in this case, we can show that the only additional cases are $\mathbb{P}(\mathcal{O}(e) \oplus \mathbf{1})$-bundles over $S^{2}$.

We conclude that there exists either an isomorphism between $H_{S^{1}}^{*}(M, \mathbf{Z})$ and $H_{S^{1}}^{*}\left(\left(S^{2}\right)^{3}, \mathbf{Z}\right)$, in which case the equivariant cohomology ring is

$$
H_{S^{1}}^{*}(M, \mathbf{Z})=\frac{\mathbf{Z}\left[a_{1}, a_{2}, a_{3}, y\right]}{\left(w_{i} a_{i} y-a_{i}^{2}\right)},
$$

or between $H_{S^{1}}^{*}(M, \mathbf{Z})$ and $H_{S^{1}}^{*}(E, \mathbf{Z})$, in which case the equivariant cohomology ring is

$$
H_{S^{1}}^{*}(M, \mathbf{Z})=\frac{\mathbf{Z}\left[a_{1}, a_{2}, a_{3}, y\right]}{\left(a_{1}\left(w_{1} y+e a_{3}-a_{1}\right), a_{2}\left(w_{2} y-e a_{3}-a_{2}\right), a_{3}\left(w_{3} y-a_{3}\right)\right)},
$$

where the $a_{i}$ 's correspond to $\alpha, \beta, \gamma$, the $w_{i}$ 's are the negative isotropy weights of the corresponding fixed points of index 2 , and $e$ is such that $\omega_{2}=\omega_{1}+e \omega_{3}$.

Note that,when the isotropy weights $q, r, p \in\{n, m, k\}$ satisfy $q \neq r$ $(\bmod p)$ only the first case is possible and then, like in the semifree case ([T-W1]), a Theorem of Wall shows that $M$ (being simply-connected) is diffeomorphic to $S^{2} \times S^{2} \times S^{2}$.

\section{References}

[A] M. Audin, The topology of torus actions on symplectic manifolds, Prog. Math. 93, Birkhäuser, Basel, 1991.

[A-B] M. Atiyah and R. Bott, The moment map and equivariant cohomology, Topology 23 (1984), 1-28.

[A-H] K. Ahara and A. Hattori, 4-dimensional symplectic $S^{1}$-manifolds admitting moment map, J. Fac. Sci. Univ. Tokyo Sect. IA, Math. 38 (1991).

[B-G-V] N. Berline, E. Getzler and M. Vergne, Heat kernels and Dirac operators, Springer-Verlag, Berlin, 1992. 
[G] R. F. Goldin, An effective algorithm for the cohomology ring of symplectic reductions, Geom. Anal. Funct. Anal. 12 (2002), 567-583.

[K] Y. Karshon, Periodic Hamiltonian flows on four dimensional manifolds. Mem. Amer. Math. Soc. 141 (1999), no. 672.

[Ki] F. C. Kirwan, The cohomology of quotients in symplectic and algebraic geometry, Princeton University Press, 1984.

[MD-S] D. McDuff and D.A. Salamon, Introduction to symplectic topology, Oxford University Press, 1995.

[MD] D. McDuff, The moment map for circle actions on symplectic manifolds, J. Geom. Phys., 5 (1988), 149-60.

[T-W1] S. Tolman and J. Weitsman, On semifree symplectic circle actions with isolated fixed points, Topology, 39 (2000), 299-309.

[T-W2] S. Tolman and J. Weitsman, The cohomology rings of Abelian symplectic quotients, Comm. Anal. Geom. 11 (2003), no. 4.

[W] C. Wall, Classification problems in differential topology $V$ : On certain 6-manifolds, Invent. Math., 1 (1966), 355-374.

Departamento de Matemática

Instituto Superior TÉCNICO

Av. Rovisco Pais

1049-001 LisBoa

PORTUGAL

E-mail address: lgodin@math.ist.utl.pt

I would like to express my most sincere gratitude to Dusa McDuff and Michèle Audin for encouragement and support as well as for all the careful explanations and comments during the preparation of this work. I am also grateful to Susan Tolman for her many helpful conversations from which this work has greatly benefited. Partially supported by FCT through program POCTI/FEDER and grants POCTI/1999/ MAT/33081 and POCTI/MAT/57888/2004, and by Fundação Calouste Gulbenkian. 
